University of Nebraska - Lincoln

DigitalCommons@University of Nebraska - Lincoln

Comprehensive Dual Liquid Chromatography with Quadruple Mass Spectrometry $($ LC1MS2 $\times$ LC1MS2 = LC2MS4) for Analysis of Parinari Curatellifolia and Other Seed Oil Triacylglycerols

William C. Byrdwell

USDA-ARS, c.byrdwell@ars.usda.gov

Follow this and additional works at: https://digitalcommons.unl.edu/usdaarsfacpub

Byrdwell, William C., "Comprehensive Dual Liquid Chromatography with Quadruple Mass Spectrometry $($ LC1MS2 $\times$ LC1MS2 = LC2MS4) for Analysis of Parinari Curatellifolia and Other Seed Oil Triacylglycerols" (2017). Publications from USDA-ARS / UNL Faculty. 1772.

https://digitalcommons.unl.edu/usdaarsfacpub/1772

This Article is brought to you for free and open access by the U.S. Department of Agriculture: Agricultural Research Service, Lincoln, Nebraska at DigitalCommons@University of Nebraska - Lincoln. It has been accepted for inclusion in Publications from USDA-ARS / UNL Faculty by an authorized administrator of DigitalCommons@University of Nebraska - Lincoln. 


\title{
Comprehensive Dual Liquid Chromatography with Quadruple Mass Spectrometry (LC1MS2 $\times$ LC1MS2 = LC2MS4) for Analysis of Parinari Curatellifolia and Other Seed Oil Triacylglycerols
}

\author{
William C. Byrdwell*i)
}

Food Composition and Methods Development Lab, Agricultural Research Service, Beltsville Human Nutrition Research Center, USDA, 10300 Baltimore Avenue, Building 161, Beltsville, Maryland 20705, United States

\section{Supporting Information}

\begin{abstract}
Online two-dimensional (2D) comprehensive liquid chromatography $(\mathrm{LC} \times \mathrm{LC})$ has become increasingly popular. Most LC $\times$ LC separations employ one or more detectors at the outlet of the second dimension, ${ }^{2} \mathrm{D}$, with very short runs to avoid undersampling. We used six detectors, including dual parallel mass spectrometry (LC1MS2), for detection of the first dimension, ${ }^{1} \mathrm{D}$. We made an argentation (silver-ion) UHPLC column from a strong cation exchange column for ${ }^{2} \mathrm{D}$, coupled with $\mathrm{UV}$ and LC1MS2 detection. LC1MS2 in ${ }^{1} \mathrm{D}$ combined with LC1MS2 in ${ }^{2} \mathrm{D}$, plus five other detectors, constituted LC2MS4 in a comprehensive LC1MS2 $\times$ LC1MS2 2D-LC separation. Electrospray ionization (ESI) high resolution accurate mass (HRAM) mass spectrometry

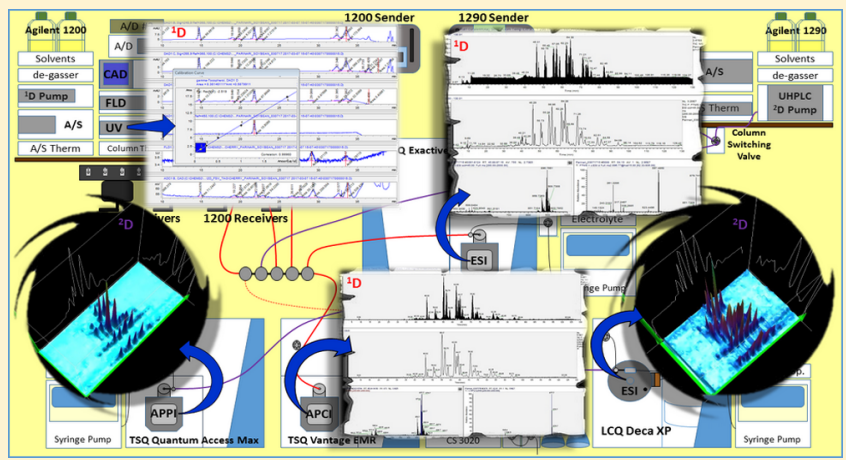
(MS) and atmospheric pressure chemical ionization (APCI) MS were used in parallel for ${ }^{1} \mathrm{D}$ detection, while atmospheric pressure photoionization (APPI) MS and ESI-MS were used for detection of ${ }^{2} \mathrm{D}$. The LC1MS2 used for ${ }^{1} \mathrm{D}$ allowed quantification of triacylglycerol (TAG) molecular species of Parinari curatellifolia and other seed oils, while the ${ }^{2} \mathrm{D}$ allowed isomers of TAG containing 18:3 fatty acyl chains as well as TAG regioisomers to be separated and identified. The LC1MS2 in ${ }^{1} \mathrm{D}$ allowed identification of oxo-TAG species by HRAM MS and quantification of $806.3 \pm 1.3$ and $1101 \pm 22 \mu \mathrm{g} / \mathrm{g}$ of $\alpha$ - and $\gamma$ tocopherols, respectively, in P. curatellifolia by APCI-MS. It is now feasible to use silver-ion UHPLC as the ${ }^{2} \mathrm{D}$ separation in LC $\times$ $\mathrm{LC}$ and to use multiple mass spectrometers across both dimensions to perform conventional quantitative analysis and to take advantage of the newest LC $\times$ LC separation technology to identify isomers that are otherwise difficult to separate.
\end{abstract}

$\mathrm{N}$ umerous reviews of two-dimensional liquid chromatography (2D-LC) techniques have appeared in recent years, with those covering basic theory and principles ${ }^{1-3}$ and describing 2D-LC coupled to mass spectrometry ${ }^{4}$ (MS) being especially useful for the work described herein. Excellent chapters describing both theoretical and practical aspects with citations for numerous reviews and applications in a wide range of fields have recently appeared. ${ }^{5,6}$ Note that the nomenclature of Marriott et al. ${ }^{7}$ and Schoenmakers et al., ${ }^{8}$ as reflected in the chapter by Stoll, ${ }^{6}$ is used here. Although the peak capacity in LC $\times$ LC is theoretically multiplicative (the product of the $1 \mathrm{D}$ LC peak capacities) if the $2 \mathrm{D}$ separations are perfectly orthogonal, but in practice the maximum theoretical peak capacity is rarely achieved. ${ }^{6}$ Nevertheless, it is typically possible to achieve a higher (often much higher) peak capacity by employing 2D-LC rather than 1D-LC.

Conventional LC $\times \mathrm{LC}$ is typically done by using a low flow rate in the ${ }^{1} \mathrm{D}$, which is all directed to the ${ }^{2} \mathrm{D}$. The low flow rate helps minimize solvent incompatibility with the ${ }^{2} \mathrm{D}$ solvent system and provides wider peaks to allow more fractions to be taken across the ${ }^{1} \mathrm{D}$ peaks, which minimizes undersampling and limits the sample amount on the ${ }^{2} \mathrm{D}$ column to facilitate peak refocusing. $2 \mathrm{D}$-LC often uses very high flow rates in the ${ }^{2} \mathrm{D}$ to provide very fast runs so that several $2 \mathrm{D}$ runs can be accomplished over the width of a 1D peak to adequately reconstruct the peak profile. As Davis, Stoll, and Carr 9 discussed elsewhere, undersampling results when too few samples are taken across a peak.

Mondello and co-workers ${ }^{10-12}$ have pioneered the use of comprehensive LC $\times$ LC for triacylglycerols (TAG) using Agion chromatography, which does a partial separation into groups by degree of unsaturation coupled to nonaqueous reversed-phase (NARP) HPLC, which further separates into distinct peaks by partition number $(\mathrm{PN})$, where the $\mathrm{PN}=$ \# carbons $-2 \times \#$ double bonds. Their work included the use of atmospheric pressure chemical ionization (APCI) MS for detection. Others soon followed with solvent modifications, etc., aimed at providing improved separations, ${ }^{13}$ and a variety of lipid applications. ${ }^{14-18}$

Received: July 14, 2017

Accepted: August 29, 2017

Published: August 29, 2017 
One thing that most methods for TAG analysis by 2D-LC have in common is the use of silver-ion chromatography in the ${ }^{1} \mathrm{D}$, followed by NARP UHPLC in the ${ }^{2} \mathrm{D}$. This is due in large part to the commercial availability of silver-ion HPLC columns and the lack of availability of silver-ion UHPLC columns (and even a dearth of strong cation exchange (SCX) UHPLC columns from which to make silver-ion columns). Unfortunately, Ag-ion HPLC produces clusters of peaks, with TAGs having similar degrees of unsaturation appearing in only partially resolved clusters. This puts a greater demand on the ${ }^{2}$ D NARP-UHPLC separation to resolve those clusters.

In contrast, we already had a NARP-HPLC separation ${ }^{19}$ that we liked for 1D-LC and just wanted to use Ag-ion UHPLC to tease apart a few remaining overlaps and separate isomers. Therefore, we made our own silver-ion UHPLC column from one of the few SCX columns available, which we loaded with silver using an approach that differs from the classic approach that is still commonly used. ${ }^{20}$ Our standard NARP-HPLC method $^{19}$ utilized a substantial amount of ACN, which caused a lack of retention on the silver-ion UHPLC column. Thus, we implemented a methanol/ethanol/dichloromethane $(\mathrm{MeOH} /$ $\mathrm{EtOH} / \mathrm{DCM}$ ) gradient for NARP-HPLC that is compatible with the Ag-ion second dimension. The resolution is not quite as good as our standard $\mathrm{MeOH} / \mathrm{ACN} / \mathrm{DCM}$ method, but this is compensated for by the separation in the ${ }^{2} \mathrm{D}$.

Due to our earlier interest in the $\alpha$-eleostearic acid ( $\alpha$-EA) $(9 Z, 11 E, 13 E$-octadecatrienoic acid)-containing TAGs in the seed oil of cherry (Prunus cerasus) pit oil (CPO), the new approach was applied to the same CPO and to parinari (African Mobola Plum, Parinari curatellifolia) seed oil (PSO) and wild soybean (Glycine soja) oil (SBO). Primary emphasis is on PSO because, in addition to general nutrition parameters ${ }^{21,22}$ (protein, fiber, moisture, ash, total fat), little more than the fatty acid (FA) composition has been previously reported, ${ }^{21}$ with that being erroneous or incomplete, although some limited data for related species are also reported. There has been reference to $\alpha$-EA in other parinari species, ${ }^{23-25}$ which contributed to our interest in the samples reported here. We also extend our previous approach ${ }^{19}$ beyond vitamin $\mathrm{D}$ analysis to other fat-soluble vitamins, specifically tocopherols in all three seed oils.

We report here the first demonstration of comprehensive 2D-LC with double dual parallel mass spectrometry. Two mass spectrometers operated in APCI-MS and ESI-high resolution accurate mass (HRAM)-MS modes as well as UV, a fluorescence detector (FLD), corona charged aerosol detector (CAD), and an evaporative light scattering detector (ELSD) were used to monitor the first dimension, coupled with two other mass spectrometers operated in atmospheric pressure photoionization (APPI)-MS and ESI-MS modes plus UV, for a comprehensive LC2MS4 (or LC1MS2 $\times$ LC1MS2) analysis. This approach allowed the first report of the diacylglycerol (DAG) and TAG composition of PSO, identification of a previously unreported oxo-FA, and the first report and quantification of tocopherols in PSO and improved analysis of tocopherols in CPO. Results were supplemented and confirmed by gas chromatography (GC) with a flame ionization detector (FID) and GC-MS in electron impact (EI) and chemical ionization $(\mathrm{CI})$ modes.

\section{EXPERIMENTAL SECTION}

Due to the number of instruments used for LC2MS4, most instrument details are provided in the Supporting Information.

Column Preparation. An Epic-SCX strong cation exchange $(\mathrm{SCX})$ column, $100 \times 2.1 \mathrm{~mm}, 3 \mu \mathrm{m}$ particles (\#122191-ESCX), was obtained from ES Industries, Inc. An old Constametric 4100 MS quaternary HPLC pump was used to flush the SCX column with Millipore D.I. $\mathrm{H}_{2} \mathrm{O}$ for at least an hour at $0.2 \mathrm{~mL} / \mathrm{min}$. Then, a $500 \mathrm{~mL}$ bottle of $1.0 \mathrm{M} \mathrm{AgNO}_{3}$ solution (Sigma-Aldrich) was fitted with a cap and line and fed directly into the Y-fitting supplying the reciprocating pump heads. Initially, the outlet of the column was sent to waste; then, after $\sim 30 \mathrm{~min}$, the outlet was directed back into the $\mathrm{AgNO}_{3}$ bottle. The system was located in a room with subdued light, and the bottle of $\mathrm{AgNO}_{3}$ was placed inside two closed nested boxes to eliminate light exposure. The solution was allowed to recycle through the column overnight at $0.2 \mathrm{~mL} /$ min, which represented $\sim 450$ column volumes over $13 \mathrm{~h}$. Next, the $\mathrm{AgNO}_{3}$ was removed, and the column was flushed with D.I. $\mathrm{H}_{2} \mathrm{O}$ at $0.2 \mathrm{~mL} / \mathrm{min}$ for at least an hour. Finally, the column was flushed with $\mathrm{MeOH}$ at $0.2 \mathrm{~mL} / \mathrm{min}$ for an hour, after which it was ready for use.

2D-LC Instrumentation. An Agilent $1200 \mathrm{HPLC}$ system that employed two Inertsil ODS- 2 columns in series, $250 \times 4.6$ $\mathrm{mm}, 5 \mu \mathrm{m}$ particles, which has been described previously, ${ }^{19}$ was used for the ${ }^{1} \mathrm{D}$ separation. A fluorescence detector (FLD) was added between the diode array detector (DAD) and flow splitter since the earlier report. A splitting system controlled flow to each detector based on the length and I.D. of the fused silica capillary directed to each instrument. Full details of all components are provided in the Supporting Information and are depicted in Figure 1 . In summary, the ${ }^{1} \mathrm{D}$ was monitored

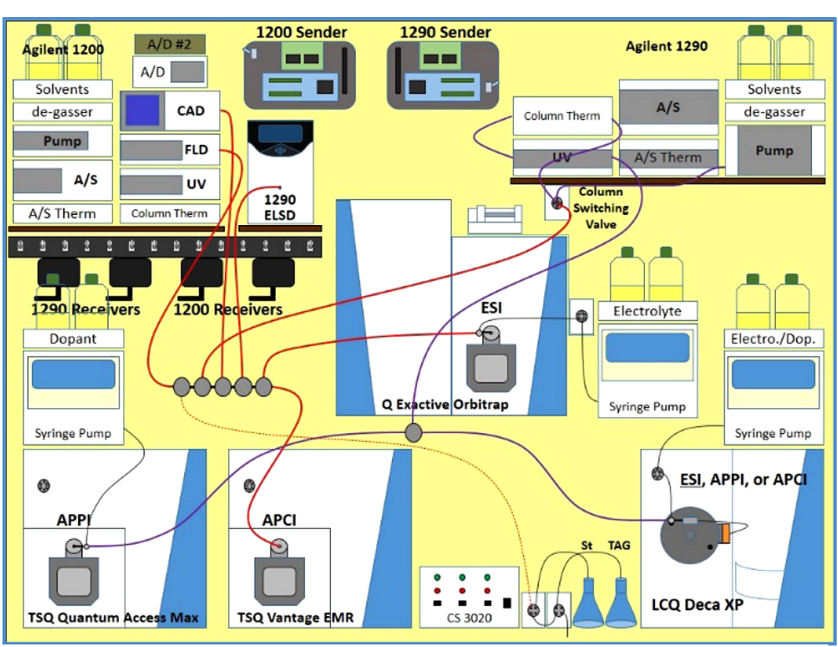

Figure 1. LC1MS4 configuration of instruments for comprehensive LC1MS2 $\times$ LC1MS2 plus UV, FLD, CAD, and ELSD.

using detection by a $\mathrm{DAD}$, the FLD, the $\mathrm{CAD}$, the ELSD (data not shown), a TSQ Vantage EMR mass spectrometer operated in APCI-MS mode, and a Q Exactive HRAM instrument operated in ESI-MS mode (with $20 \mathrm{mM} \mathrm{NH}_{4} \mathrm{OCOH}$ in $\mathrm{ACN}$, $1: 4$, at $20 \mu \mathrm{L} / \mathrm{min}$ via syringe pump). One branch of the splitter, having a flow rate of $53.67 \mu \mathrm{L} / \mathrm{min}$, was directed to the Agilent G1170A switching valve with two $100 \mu \mathrm{L}$ sample loops installed, producing a loop fill time of $1.86 \mathrm{~min}$ and modulation time of $1.91 \mathrm{~min}$. The switching valve served as the interface to 
an Agilent 1290 UHPLC system composed of a binary pump, column oven with the Epic-SCX/Ag-ion column installed, and $\mathrm{DAD}$. Flow after the DAD no. 2 was directed to a single Valco tee splitter, with the two branches going to a TSQ Quantum Access Max mass spectrometer operated in APPI-MS mode with acetone dopant supplied by a dual piston syringe pump at $50 \mu \mathrm{L} / \mathrm{min}$ and an LCQ Deca XP ion trap mass spectrometer operated in ESI mode with $50 \mu \mathrm{L} / \mathrm{min} \mathrm{NH}_{4} \mathrm{OCOH}$ via syringe pump. Syringe pumps were $\mathrm{AB} 140 \mathrm{~B} / \mathrm{C}$ dual piston syringe pumps. Syringe pumps for ESI instruments were plumbed through electronically controlled valves attached or built into the instruments to flush deionized water (from old HPLC pumps) through the sources between runs to reduce problems with clogging. Control of all instruments was coordinated using the 14-switch wireless communication contact closure system (WCCCS) previously described. ${ }^{26}$ Visualization of the 2D-LC chromatograms was done using LC Image v. $2.5 \mathrm{~b} 7$ software from GC Image, Inc.

GC Instrumentation. Analyses on an Agilent 6890N GC with a FID and an Agilent 7890A GC with 5975C MS (in EI and CI modes) were performed using the instruments and conditions recently reported. ${ }^{27}$ Column and flow conditions are given in the Supporting Information.

Quantification. Calibration levels of $0.125,0.250,0.500$, 1.00 , and $2.00 \mu \mathrm{g} / \mathrm{mL}$ were prepared from $25.0 \mu \mathrm{g} / \mathrm{mL}$ (nominal) stock solutions of each fat-soluble vitamin (FSV) listed below with each concentration adjusted for standard purity (from Certificates of Analysis) and precise stock solution concentration. $\mathrm{d}_{6}-\alpha$-Tocopherol at $1.00 \mu \mathrm{g} / \mathrm{mL}$ was added as IS to all standards and samples. Quantification of FSVs by MS was done using APCI-MS in time-segmented selected ion monitoring (SIM) and selected reaction monitoring (SRM) modes using the parameters listed in the Supporting Information. Quantification of DAGs and TAGs was done using the GC-FID response-factor-adjusted approach previously described, ${ }^{28,29}$ with inclusion of $1 \times{ }^{13} \mathrm{C}$ isotopic peaks for added sensitivity without loss of specificity, as previously discussed. ${ }^{30}$ Quantification of FSVs by UV detection was done using wavelengths adapted from Ball, ${ }^{31}$ specifically $297 \mathrm{~nm}$ for $\alpha$-, $\gamma$-, and $\delta$-tocopherols, $265 \mathrm{~nm}$ for vitamin $\mathrm{D}_{2}$ and $\mathrm{D}_{3}, 326$ $\mathrm{nm}$ for retinol (vitamin $\mathrm{A}$ ), retinyl acetate, and retinyl palmitate, and $248 \mathrm{~nm}$ for phylloquinone (vitamin $\mathrm{K}_{1}$ ). Fluorescence detection of tocopherols was done using a legacy detector (Agilent 1100 series) at $330 \mathrm{~nm}$ as a test of FLD specificity. Additional parameters for UV and FLD are given in the Supporting Information. All peaks were manually integrated, and calculations were performed using the linest() function in Excel spreadsheets using both IS and external standard approaches (ES). ${ }^{19,30}$ Because the IS was optimized for MS detection, results by UV could only be estimated as discussed below.

\section{RESULTS}

We bypassed the problem of under-sampling and problems associated with quantification of 2D-LC "blobs" by directly monitoring the ${ }^{1} \mathrm{D}$ using two mass spectrometers, operated in APCI-MS and ESI-HRAM-MS modes as well as UV, FLD, CAD, and ELSD. Chromatograms and calibration lines of $\alpha$ tocopherol by ${ }^{1}$ D SIM and SRM are shown in Figure 2. Although we did not know to expect tocopherols in PSO, we routinely run samples using our FSV and TAG screening procedure, which allows quantification of any of the FSVs mentioned above if they are present. Table 1 shows the results

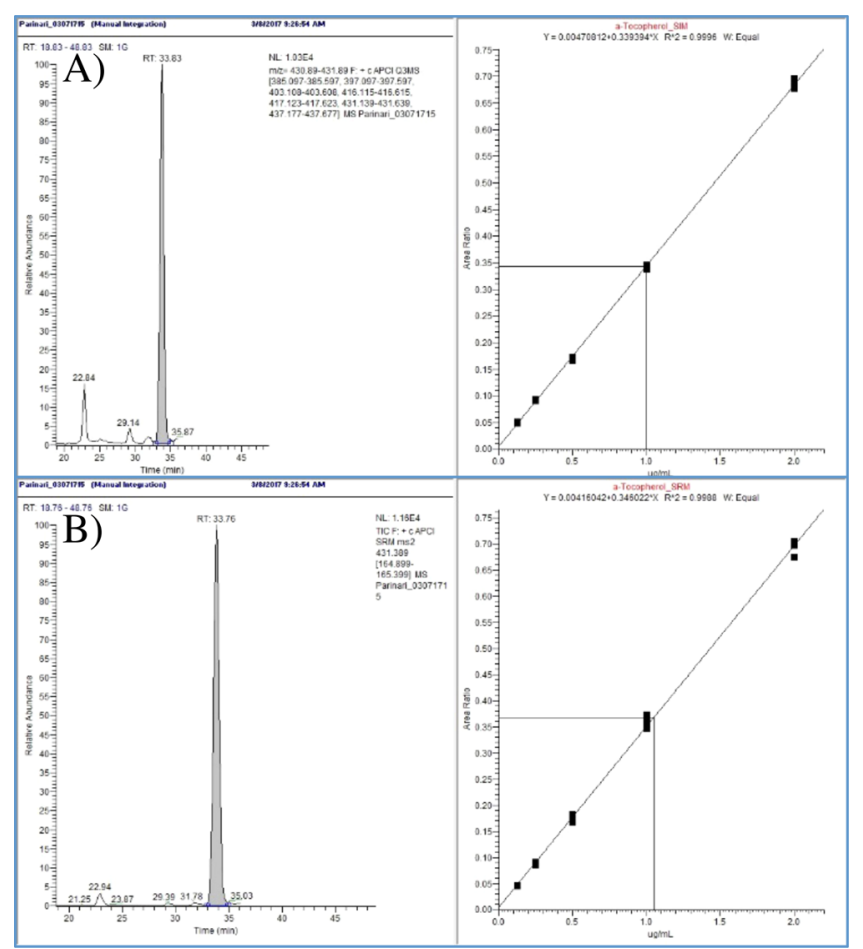

Figure 2. Chromatograms and calibration lines for $\alpha$-tocopherol by (A) selected ion monitoring and (B) selected reaction monitoring. FA abbreviations: P, palmitic acid, 16:0 (carbons:double bonds); El, $\alpha$ eleostearic acid, $9 c, 11 t, 13 t-18: 3$ ( $c=$ cis, $t=$ trans); L, linoleic acid, 18:2; O, oleic acid, 18:1; S, stearic acid, 18:0; G, gadoleic acid, 20:1; A, arachidic acid, 20:0.

Table 1. Quantification of $\alpha$-, $\gamma$-, and $\delta$-Tocopherols by SIM and SRM APCI-MS in ppm $(\mu \mathrm{g} / \mathrm{g}$ Oil $)$

\begin{tabular}{|c|c|c|c|c|c|c|}
\hline \multicolumn{7}{|c|}{ Selected ion monitoring, internal standard method } \\
\hline & $\alpha$ & SD & $\gamma$ & $\mathrm{SD}$ & $\delta$ & SD \\
\hline cherry & 293.5 & 3.5 & 630 & 26 & 97 & 14 \\
\hline parinari & 789 & 28 & 881 & 45 & 6 & 16 \\
\hline soybean & 29.9 & 0.3 & 168 & 14 & 227 & 13 \\
\hline$r^{2}$ & 0.9996 & & 0.9883 & & 0.9917 & \\
\hline \multicolumn{7}{|c|}{ Selected reaction monitoring, internal standard method } \\
\hline & $\alpha$ & SD & $\gamma$ & SD & $\delta$ & $\mathrm{SD}$ \\
\hline cherry & 281 & 12 & 897.8 & 5.4 & 120.8 & 6.9 \\
\hline parinari & 806.3 & 1.3 & 1101 & 22 & 34.0 & 6.3 \\
\hline soybean & 18.9 & 0.2 & 193.0 & 1.4 & 198 & 13 \\
\hline$r^{2}$ & 0.9988 & & 0.9943 & & 0.9956 & \\
\hline
\end{tabular}

for the IS approach by SIM and SRM APCI-MS in ppm or $\mu \mathrm{g} /$ $\mathrm{g}$ of oil $=\mathrm{mg} / \mathrm{kg}$ of oil with the first nonsignificant figure shown or to 0.1 . The coefficients of determination $\left(r^{2}\right)$ given in Table 1 indicate good linearity of the calibration lines. Results by the ES approach by APCI-MS and the IS and ES approaches by UV detection are given in the Supporting Information because these are all less desirable and reliable than the IS approach by MS. All ES and IS results by MS and UV, with the exception of UV results for $\alpha$-tocopherol in PSO, were in good to excellent agreement among all approaches. To derive an estimation of IS results by UV required approximation of the IS integrated areas for $1.00 \mu \mathrm{g} / \mathrm{mL}$ as follows: the area for each $\alpha$-tocopherol calibration standard was divided by the $\alpha$-tocopherol total amount to give $(\mathrm{area} /(\mu \mathrm{g} / \mathrm{mL}))$. Each area for all other FSVs was divided by the same-run normalized IS area. For samples, 
the average normalized signal area across all standards (= $5.5294 \pm 0.6330)$ was used as the IS area because it was unknown how much of the $\alpha$-tocopherol was attributable to the IS and how much came from the oil sample. Again, no such approximation was required for results by ES or IS by MS or ES by UV.

FAs, DAGs, and TAGs. The FA composition calculated from the sum of response-factor-normalized DAGs and TAGs and by GC-FID for FAs present at $\geq 0.1 \%$ for P. curatellifolia is given in Table 2. Due to space limitations, all 18:3 species are

Table 2. FA Composition Calculated from DAG and TAG Composition of $\boldsymbol{P}$. cuatellifolia Compared to FA from GCFID of FA Methyl Esters

\begin{tabular}{lccc}
\multicolumn{1}{c}{ FA } & average (\%) & SD (\%) & GC-FID (\%) \\
P & 9.1 & 0.0 & 9.3 \\
El & 48.8 & 0.1 & 48.8 \\
L & 14.4 & 0.1 & 14.0 \\
O & 17.7 & 0.1 & 17.5 \\
S & 7.3 & 0.1 & 7.6 \\
A & 0.4 & 0.0 & 0.4 \\
G & 0.7 & 0.0 & 0.7 \\
oxo-El & 1.6 & 0.0 & 1.5 \\
sum & 99.9 & & 99.9 \\
\hline
\end{tabular}

grouped together in Table 2, as are all oxo-18:3 FA. A more detailed composition is provided in the Supporting Information. As identified by GC-MS and quantified by GC-FID, 90.0 $\pm 0.1 \%$ of $18: 3$ was $\alpha$-eleostearic acid, $7.6 \pm 0.1 \%$ was $\beta$-El, a third isomer (unidentified) represented $1.7 \pm 0.0 \%$, and only $0.1 \pm 0.0 \%$ was normal $\mathrm{Ln}$, and these comprise almost all of the $48.8 \%$ in Table 2. While eleostearic acid has been reported previously, this represents the first report of oxo-eleostearic acid in P. curatellifolia. Four oxo-El isomers were found by GC-MS and quantified by GC-FID, with the two major isomers representing $81.0 \pm 0.8 \%$ and $13.3 \pm 0.3 \%$, comprising the majority of the $1.5 \%$ of oxo-El shown in Table 2. The compositions of DAGs and TAGs are given for the first time in Tables 3 and 4, respectively. DAGs represented only $1.52 \%$ of

Table 3. Response Factor Normalized DAG Composition for P. curatellifolia

$\begin{array}{lccc}\text { DAG } & \text { RT }(1)^{a} & \text { \% comp } & \text { DAG pk. 2/1 }{ }^{b} \\ \text { oxElEl } & 19.09 & 0.7 & 0.21 \\ \text { oxElL } & 21.09 & 0.1 & 0.28 \\ \text { oxElO } & 26.30 & 0.2 & 0.23 \\ \text { oxElP } & 26.59 & 0.1 & 0.30 \\ \text { ElEl } & 32.46 & 17.4 & 2.57 \\ \text { PoO } & 35.68 & 0.8 & 1.61 \\ \text { LEl } & 35.84 & 11.2 & 2.72 \\ \text { LL } & 37.60 & 11.7 & 4.19 \\ \text { OEl } & 39.02 & 12.5 & 1.57 \\ \text { OL } & 39.77 & 14.7 & 3.43 \\ \text { PL } & 39.96 & 6.8 & 1.53 \\ \text { OO } & 41.73 & 6.9 & 1.50 \\ \text { OP } & 42.00 & 7.0 & 0.72 \\ \text { SL } & 42.55 & 4.8 & 1.59 \\ \text { OS } & 44.99 & 5.1 & 0.52 \\ \text { sum } & 99.9 \% & & \end{array}$

${ }^{a}$ Retention time for first peak of the pair. ${ }^{b}$ Ratio of DAG peak 2 to DAG peak 1 .
Table 4. Response Factor Normalized TAG Composition for P. curatellifolia

\begin{tabular}{|c|c|c|c|c|c|}
\hline TAG & RT & $\%$ comp & TAG & RT & $\%$ comp \\
\hline oxElElEl & 46.02 & 1.3 & POL & 75.30 & 2.1 \\
\hline oxElElL & 47.08 & 0.4 & LLS & 76.15 & 0.5 \\
\hline oxElElO & 50.54 & 0.9 & PPL & 77.15 & 0.7 \\
\hline oxElElP & 51.23 & 0.7 & LElA & 79.48 & 0.3 \\
\hline oxElLO & 51.87 & 0.1 & ElElA & 79.89 & 0.5 \\
\hline oxElOO & 56.21 & 0.1 & SOEl & 82.19 & 3.0 \\
\hline oxElElS & 56.26 & 0.9 & $\mathrm{OOO}$ & 82.21 & 1.0 \\
\hline oxElOP & 56.83 & 0.1 & OLG & 82.29 & 0.3 \\
\hline oxElOS & 63.36 & 0.1 & OOP & 84.24 & 1.4 \\
\hline ElElEl & 55.11 & 12.0 & ElSP & 84.32 & 1.0 \\
\hline ElElL & 56.70 & 8.3 & PLG & 84.37 & 0.2 \\
\hline LLEl & 58.47 & 3.3 & SLO & 85.04 & 1.4 \\
\hline LLL & 60.31 & 0.4 & POP & 86.29 & 0.5 \\
\hline ElElO & 62.05 & 11.3 & SLP & 87.09 & 0.9 \\
\hline ElElP & 63.23 & 8.1 & OOG & 90.98 & 0.1 \\
\hline OLEl & 64.10 & 7.8 & ElOA & 91.46 & 0.1 \\
\hline PLEl & 65.44 & 4.6 & oOs & 93.68 & 0.8 \\
\hline LLO & 66.33 & 1.0 & ElSS & 93.83 & 0.9 \\
\hline LLP & 67.64 & 0.9 & POS & 95.79 & 0.7 \\
\hline ElElG & 68.65 & 1.3 & PLA & 96.36 & 0.1 \\
\hline ElElS & 70.83 & 8.9 & SSL & 96.38 & 0.4 \\
\hline OOEl & 70.94 & 4.2 & PPS & 99.31 & 0.1 \\
\hline POEl & 72.50 & 3.0 & POA & 104.09 & 0.1 \\
\hline LLG & 73.27 & 0.2 & SSO & 104.19 & 0.3 \\
\hline OOL & 73.53 & 1.8 & \multicolumn{2}{|c|}{ sum } & 99.3 \\
\hline PPEl & 74.35 & 0.4 & \multicolumn{2}{|c|}{ oxo-TAG } & 4.65 \\
\hline
\end{tabular}

the total area of DAGs and TAGs. Figure 3 shows an ESIHRAM-MS chromatogram and mass spectra of eleostearic acid and two oxygen functional group containing TAGs, and Figure 4 shows the APCI-MS data acquired in parallel. The $[M$ $\left.+\mathrm{NH}_{4}\right]^{+}$and $[\mathrm{M}+\mathrm{H}]^{+}$ions for several TAGs had unexpected masses that were 14 mass units higher than those of normal eleostearic acid containing TAGs, with accompanying $[\mathrm{DAG}]^{+}$ fragments at $\mathrm{m} / z 609.450\left([\mathrm{ElEl}+14]^{+}\right)$. This mass difference could represent either a branched methyl-containing FA $(-\mathrm{H}+$ $\left.\mathrm{CH}_{3}=14.0157\right)$ or an oxo-FA $(-2 \mathrm{H}+\mathrm{O}=13.9793)$. Direct detection by HRAM ESI-MS in the ${ }^{1} \mathrm{D}$ allowed unambiguous differentiation of these two possibilities. The mass accuracy for normal, known TAGs were in the 2-4 ppm range, while for the possible methylated TAGs, the mass differences were $42-45$ ppm for the $\left[\mathrm{M}+\mathrm{NH}_{4}\right]^{+}$peaks and 62-63 ppm for the $\mathrm{m} / z$ $609.450[\mathrm{DAG}]^{+}$fragment. On the other hand, the calculated accurate masses for the oxo-eleostearic TAGs were within the 2-4 ppm range for all $\left[\mathrm{M}+\mathrm{NH}_{4}\right]^{+}$and $[\mathrm{DAG}]^{+}$fragments (normal and ox-El), providing very strong evidence that the unknown TAG molecular species contained oxo-eleostearic acid. GC-MS chromatograms (not shown) exhibited a corresponding peak at $\mathrm{m} / \mathrm{z} 306.2$ representing the oxo-18:3 FAME. Finally, the chromatographic behavior of oxo-TAGs, which eluted prior to normal TAGs on the RP-HPLC column due to increased polarity, is consistent with expected behavior under RP-HPLC conditions. Several steps were taken to confirm that the oxo-TAGs were endogenous native species and were not formed during extraction. All data confirm the identification of multiple DAG and TAG molecular species containing oxo-eleostearic acid, shown in Tables 3 and 4, with oxo-TAGs being $4.65 \%$ of the response-factor-normalized TAG integrated area. The exact type and location of the oxo- 


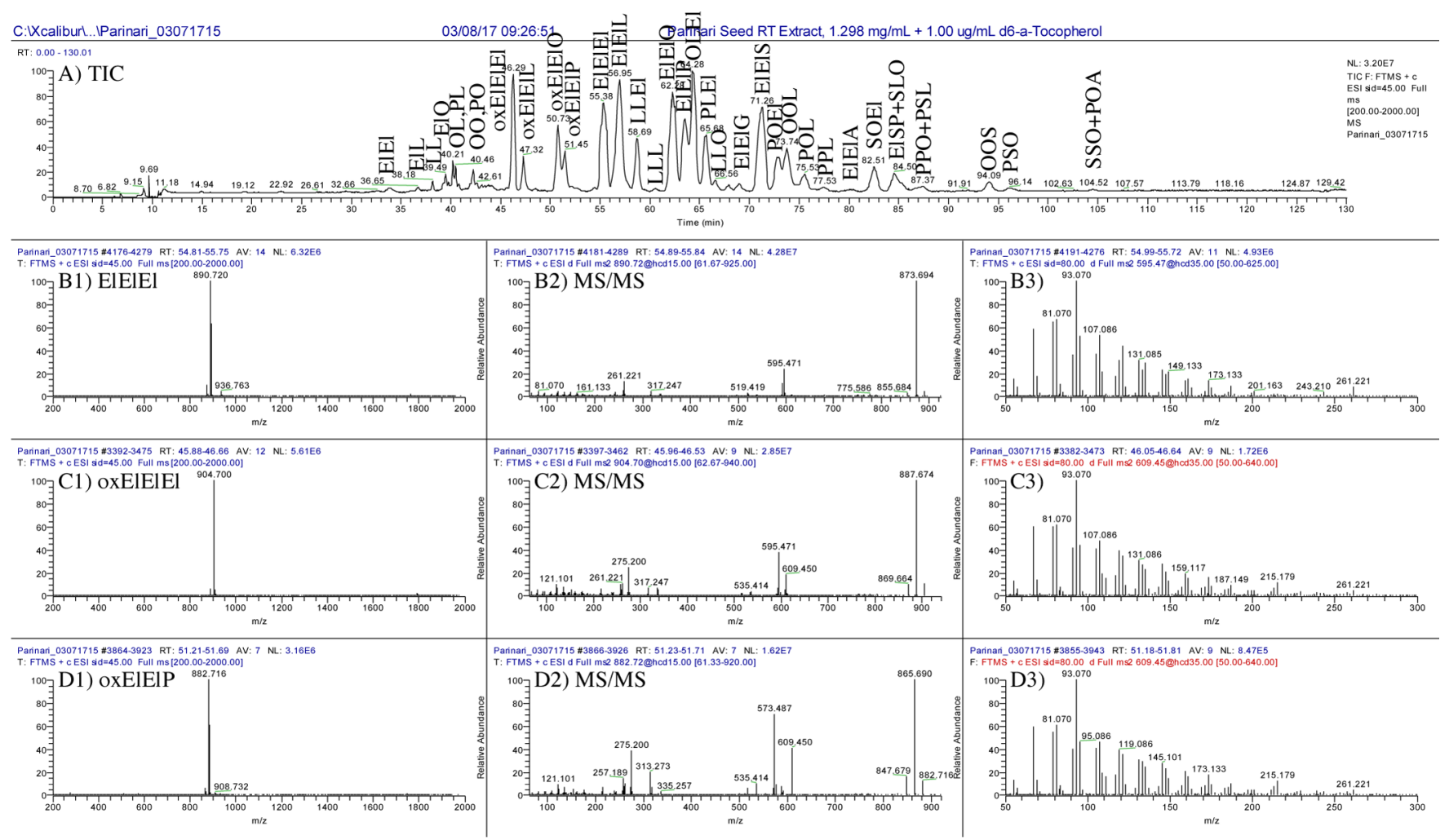

Figure 3. Q Exactive Orbitrap ESI-HRAM-MS total ion current chromatogram (TIC) and mass spectra for trieleostearin, ElElEl, and two TAGs containing previously unidentified oxo-eleostearic acid, oxElElEl, and oxElElP (column 1); low-energy CID MS/MS of $\left[\mathrm{M}^{+} \mathrm{NH}_{4}\right]^{+}($column 2); and

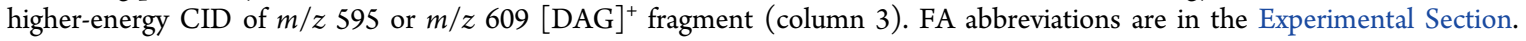

functional group could not be determined from the mass spectra alone. However, comparison of the MS/MS spectra from ElElEl and oxElElEl in Figure 3 hint that the oxo-group is not at the distal end of the FA chain, and differences in the $m / z$ 105-111 $\left(=\mathrm{C}_{8} \mathrm{H}_{x}\right)$ and $m / z 117-119\left(=\mathrm{C}_{9} \mathrm{H}_{x}\right)$ peaks indicate the possibility that it is an 8,9-oxo group. Unfortunately, time, resources, and stakeholder interest will not allow us to pursue identification of the exact identity of this oxo-FA. The early eluting peaks should be collected and subjected to IR and NMR analysis. We will be happy to share all LC-MS data. Finally, there was both HRAM ESI-MS $\left(\left[\mathrm{M}+\mathrm{NH}_{4}\right]^{+}=\mathrm{m} / \mathrm{z} 918.681\right.$ observed) and APCI-MS $\left([\mathrm{M}+\mathrm{H}]^{+}=m / z 901.6\right.$ obs $)$ evidence for the dioxo TAG oxEloxElEl present at a very low level at earlier retention time, which was not quantified.

The FA, DAG, and TAG compositions for cherry pit and wild SBO are given in the Supporting Information because these have been reported previously and were used for verification of the new approach. All results for CPO are in good agreement with the results reported recently using $1 \mathrm{D}$ NARP-HPLC. ${ }^{27}$ The SBO FA, DAG, and TAG compositions are similar to results reported elsewhere, although previous reports focused primarily on Glycine max commercially produced soybeans rather than the wild type.

Second-Dimension ( $\left.{ }^{2} D\right)$ Data. A contour plot for $P$. curatellifolia by silver-ion UHPLC with APPI-MS detection is shown in Figure 5 and that by ESI-MS is shown in Figure 6. Corresponding 3D plots are given in Figures 7 and 8. As usual, ESI-MS exhibits sensitivity greater than that of either APCI-MS or APPI-MS, giving larger peaks in Figures 6 and 8. No attempt was made to quantify the DAGs and TAGs using the contour plots because these are known to be problematic for quantification, ${ }^{6,32-35}$ and very effective quantification using a well-established approach was provided by the ${ }^{1} \mathrm{D}$ APCI-MS data. The ${ }^{2} \mathrm{D}$ data were used only for qualitative analysis. The silver-ion UHPLC column provided a separation based on the well-known principles that have been described for argentation chromatography in the past. Boryana Nikolova-Damyanova and William W. Christie provide an excellent tutorial for argentation chromatography at the Lipid Library (http://lipidlibrary.aocs. org/content.cfm?ItemNumber=40341). Because cis double bonds produce stronger complexes with silver ions than trans double bonds, ${ }^{36}$ the UHPLC column described here very effectively separated ElElEl containing all $\alpha$-eleostearic acid from ElElEl containing a $\beta$-eleostearic acid FA, as seen in Figures 5 and 6 . Thus, comprehensive NARP-HPLC $\times$ Ag-ionUHPLC appears to be well-suited for differentiating cis/trans isomers in TAGs.

Also, the position of the unsaturated FAs in TAGs has a primary influence on the retention on the Ag-ion UHPLC column, with those in the outer positions, i.e. 1 and 3, having a stronger effect on retention than those in the middle, sn-2, position. For example, Figure 9 shows a ${ }^{2}$ D UHPLC ESI-MS EIC for $m / z$ 904.8, which represents multiple isobaric TAG molecular species and their isomers.

First, this shows that most peaks eluted in single, unsplit peaks, although some (e.g., OSO) straddled two modulation periods separated by the modulation time, as shown for OSO in Figure 9. This greatly simplified interpretation of data and gave the sharp peaks exemplified in Figures 7 and 8. Second, the silver-ion column readily differentiated between two oleic acid chains in the 1,2 positions in OOS from two oleic chains in the 1,3 positions in OSO. However, because retention was based on degree of unsaturation, with minimal effect of chain length, OSO and GPO, which both have two monounsaturated FAs in 

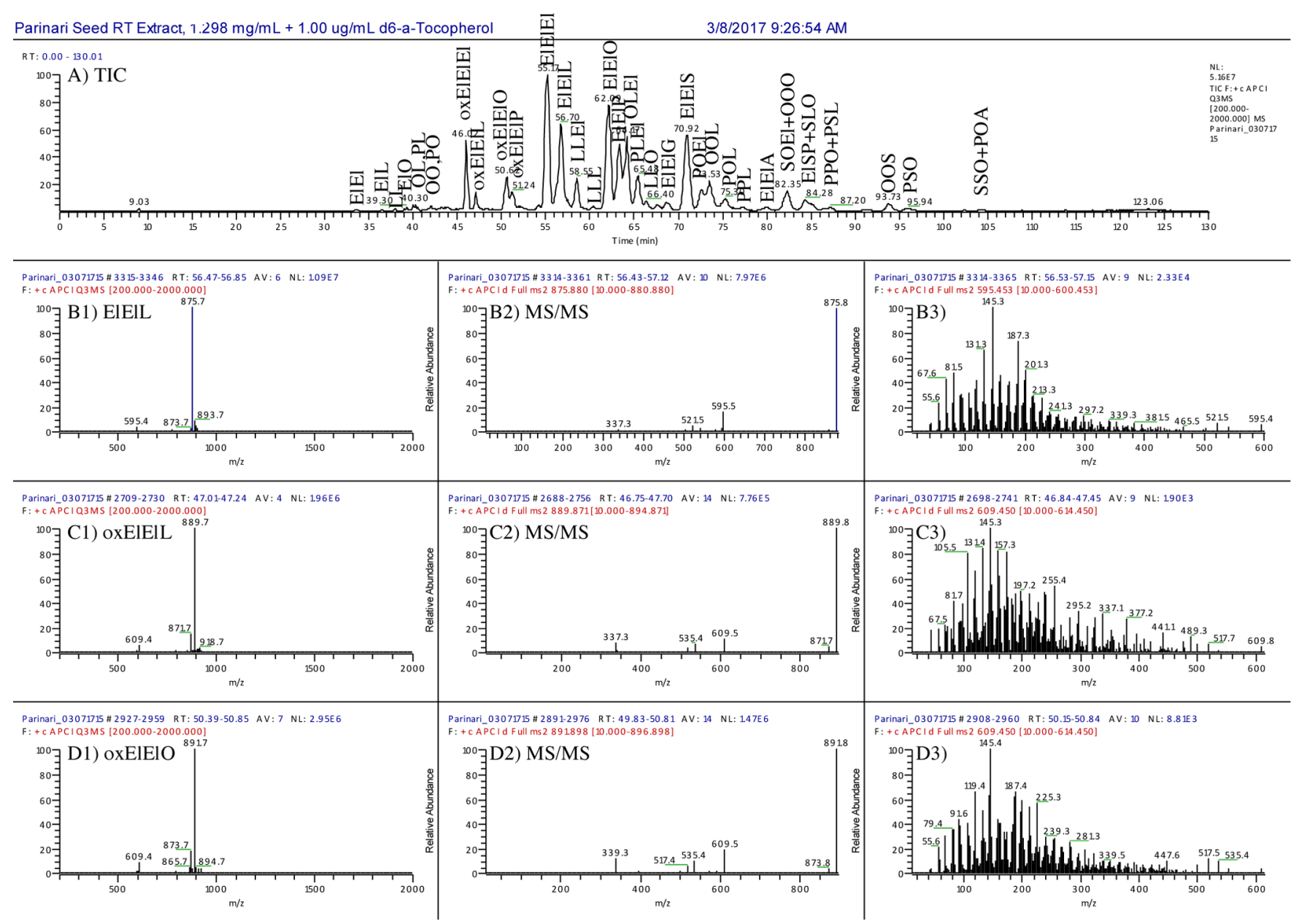

Figure 4. TSQ Vantage EMR APCI-MS TIC and mass spectra for ElElL, oxElElL, and oxElElO (column 1), low-energy CID MS/MS of $[\mathrm{M}+\mathrm{H}]^{+}$

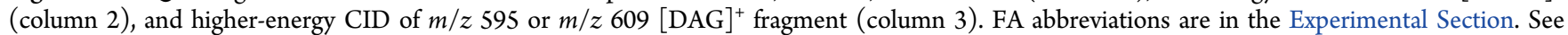
Table 4 for TAG composition from APCI-MS.

the 1,3 positions were not separated, although these could be distinguished by the different $[\mathrm{DAG}]^{+}$fragments despite having one $[\mathrm{DAG}]^{+}$fragment, $m / z 605.5$, and the protonated molecule mass in common. Similarly, SLS was differentiated from SSL due to the different position of the "L" FA. But SLS was not separated from PLA, which has different saturated FA chain lengths at 1 and 3, but the "L" FA in the same $s n$-2 position. In general, the first row of TAGs eluted in Figures 5 and 6 had one or no cis double bonds in one of the 1 or 3 positions with one or no cis double bonds in the $s n-2$ position, with the exception of oxo-El, in which the oxo- group appeared to reduce coordination of $\mathrm{El}$ with the $\mathrm{Ag}^{+}$and reduce retention (i.e., oxElElEl). The second row of TAGs had either two cis double bonds in the 1 or 3 positions or one diunsaturated FA (i.e., "L") in the $s n-2$ position. Additional unsaturation in the 1,3 positions had a greater influence on retention with additional unsaturation in the $s n-2$ position having a slightly lesser effect on retention, as mentioned above. Because APPI-MS and ESIMS were used for detection in the ${ }^{2} \mathrm{D}$, assignment of regioisomer identities by the Critical Ratio ${ }^{37,38}[\mathrm{AA}]^{+} /[\mathrm{AB}]^{+}$ by MS was less reliable ${ }^{38}$ than by APCI-MS. For instance, the difference in $[\mathrm{OO}]^{+} /[\mathrm{OS}]^{+}$between OOS sand $\mathrm{OSO}(2)$ in Figure 9 is not as large as expected or reported using APCIMS. ${ }^{39}$ Fortunately, Ag-ion UHPLC was very effective for separating regioisomers, reducing the need to rely on fragment ratios in ESI-MS/MS or APPI-MS mass spectra.

\section{DISCUSSION}

Although complete details are not given, Powell ${ }^{40}$ described producing a silver-ion column from a sulfonate derivatized silica column by flowing silver nitrate through the column. Although we were not initially aware of that work, we used a similar approach, differing primarily in the fact that we employed exhaustive saturation by recycling $\mathrm{AgNO}_{3}$ through a commercially available SCX column overnight. Both approaches contrast the more commonly used approach by Christie ${ }^{20}$ of manually injecting $\mathrm{AgNO}_{3}$ solution, which has been widely used to good effect. In the first sequence of runs immediately after preparation of a new column, small amounts of silver adducts were formed during ESI-MS, but these quickly disappeared with further use. No corrosive effects of $\mathrm{AgNO}_{3}$ elution were observed in the ionization source of any instrument. This approach has been used both for $1.8 \mu \mathrm{m}$ particle and $3.0 \mu \mathrm{m}$ particle columns, but we prefer the latter for increased robustness.

It is important to emphasize that the excellent quantitative results reported here would not be possible using conventional comprehensive $2 \mathrm{D}$-LC approaches. Quantification of ${ }^{2} \mathrm{D} 2 \mathrm{D}$ LC data is an ongoing area of development, ${ }^{32}$ but is not yet as straightforward as conventional integration of $1 \mathrm{D}$ or ${ }^{1} \mathrm{D}$ chromatograms. As mentioned by Place et al., ${ }^{33}$ each $2 \mathrm{D}$ peak consists of individual $1 \mathrm{D}$ chromatograms (slices) that 


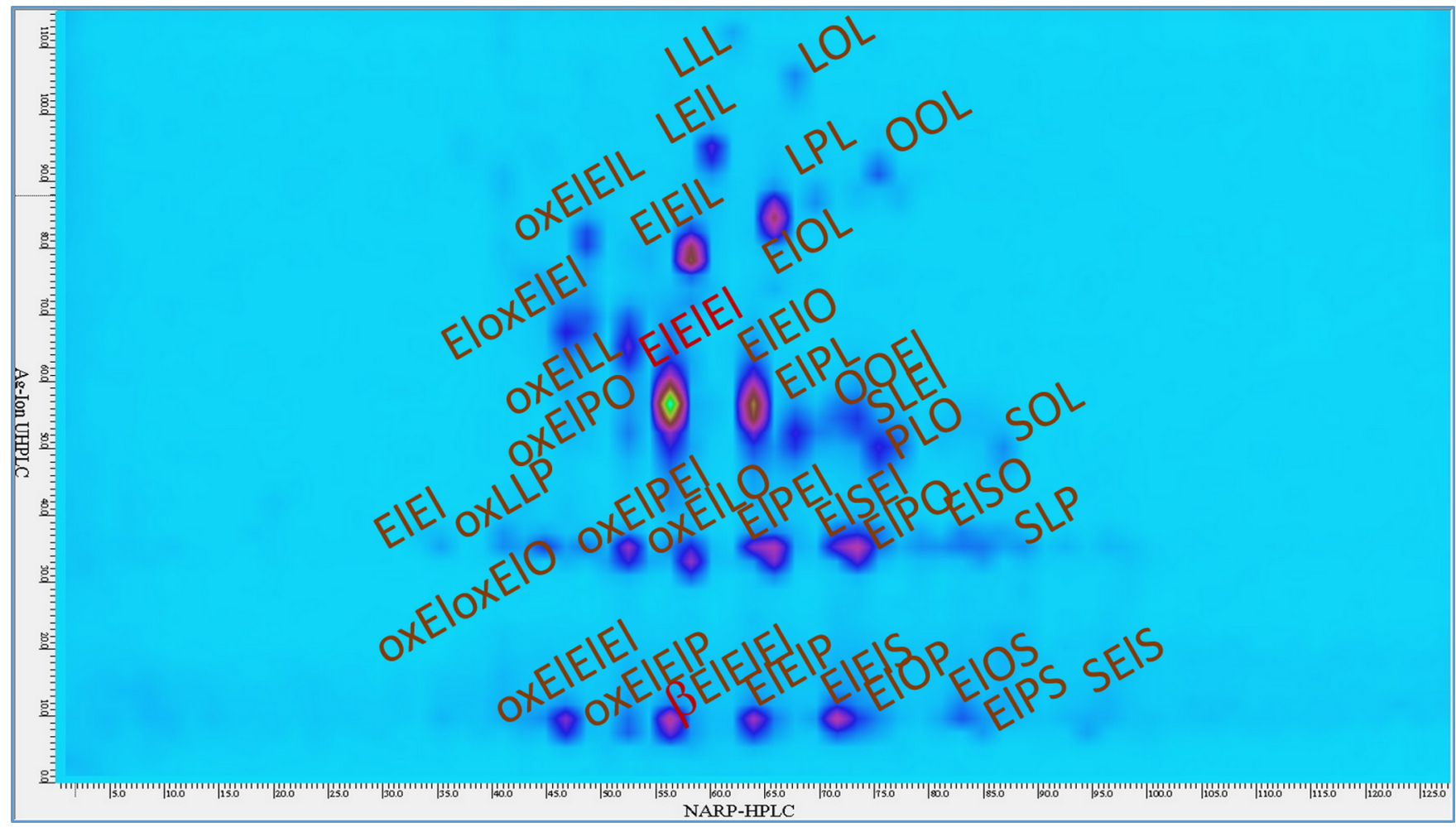

Figure 5. Second dimension silver-ion UHPLC separation with APPI-MS of P. curatellifolia seed oil.

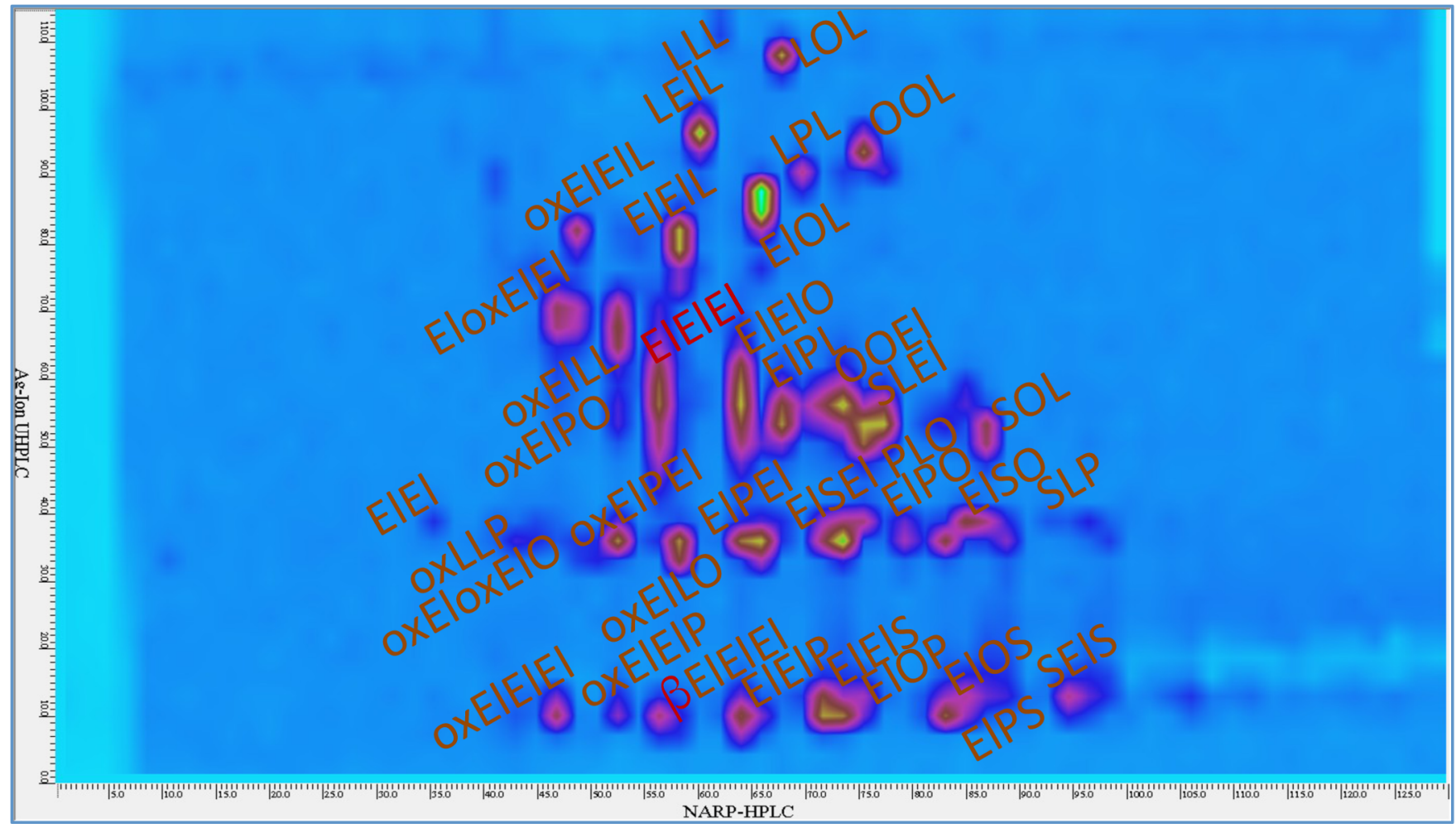

Figure 6. Second dimension silver-ion UHPLC separation with ESI-MS of P. curatellifolia seed oil.

would normally be manually integrated in $1 \mathrm{D}$ analysis. But having numerous slices across each $2 \mathrm{D}$ peak makes such integration impractical. So, they described several approaches for automated integration of $2 \mathrm{D}$ peaks, which involved labwritten procedures in the $\mathrm{R}$ programming language. Cook et al. ${ }^{34}$ discussed the several-fold higher \%RSDs encountered in 2D-LC and the reasons for these and many other factors, including the poorer $\mathrm{S} / \mathrm{N}$ due to peak dilution caused by peak fractionation. They employed a single UV detector at the outlet of the ${ }^{1} \mathrm{D}$, for $2 \mathrm{D}$ assisted LC, or 2DALC. Recently, targeted 


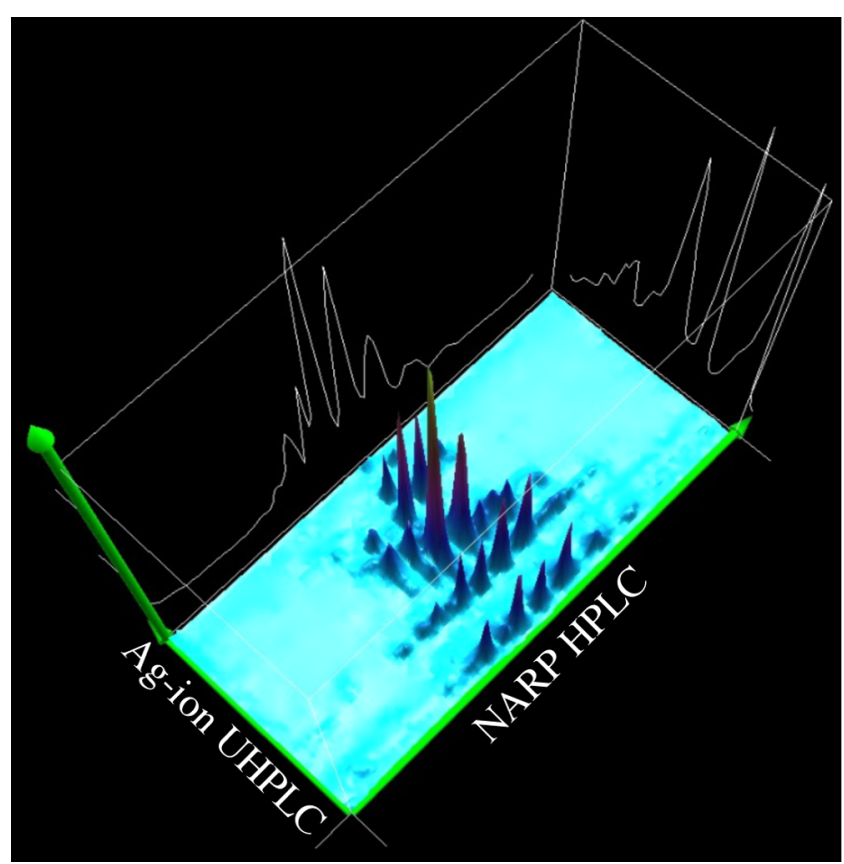

Figure 7. 3D plot of Ag-ion UHPLC APPI-MS for P. curatellifolia.

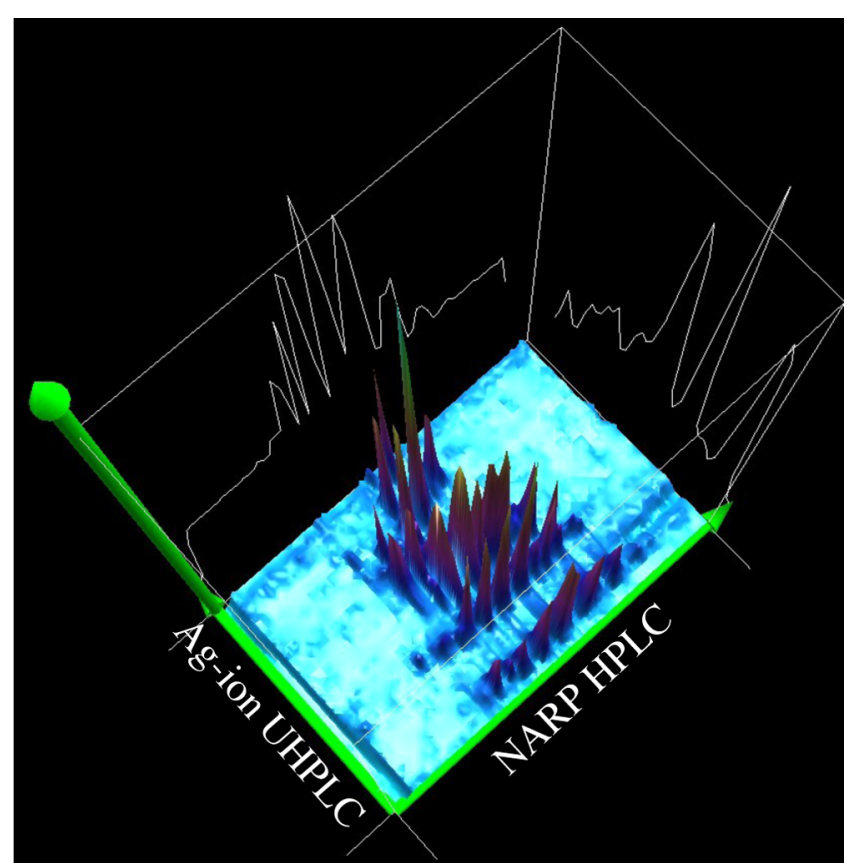

Figure 8. 3D plot of Ag-ion UHPLC ESI-MS for P. curatellifolia.

quantitative MS analysis of two target compounds using multiple reaction monitoring (MRM) was combined with qualitative analysis of wine polyphenols. ${ }^{35}$ But because we wanted to quantify multiple FSVs and semiquantify a large number of DAGs and TAGs (by relative percentage composition), we took an approach similar to 2DALC but with many more detectors.

Quantification of CPO tocopherols was included because the extract-and-shoot approach gave values that were substantially higher than those values reported by a commercial lab in our recent report. The results reported earlier were obtained using the traditional approach to sterol analysis, involving heated saponification, extraction, and collection of unsaponifiable material and derivatization followed by GC-FID analysis. By eliminating all harsh and inefficient treatment such as saponification and derivatization, we observed higher levels. Additional work is underway to confirm these results by analysis of standard reference material 3278 from the National Institute of Standards and Technology. Furthermore, the use of both SIM and SRM (often thought of as the "gold standard" for analysis) MS provided very strong confirmation of tocopherol identity as well as quantity. Additionally, by obtaining survey MS and data-dependent MS/MS scans on all instruments, we are able to refute the presence of $\beta$-sitosterol that was reported by the same commercial lab using the same saponification, extraction and collection, and derivatization approach in CPO. Using APCI-MS EICs for a $5 \mathrm{Da}$ mass range including $\gamma$ tocopherol (Table 1) $\left([\mathrm{M}+\mathrm{H}]^{+}=m / z 417.4\right)$ and $\beta$-sitosterol $\left([\mathrm{M}+\mathrm{H}]^{+}=m / z 415.4\right)$, we were able to clearly see the peaks for $\gamma$-tocopherol but not $\beta$-sitosterol. This demonstrates that peaks at a given retention time by GC without confirmation by MS, especially after extensive chemical pretreatment, are not sufficient for identification and quantification. Thus, it is advisible to view commercial lab results that do not include MS with healthy skepticism. Similar skepticism should be applied to SIM versus SRM results. Any interfering species that produce fragments or other ions similar to target compounds can skew SIM results. SRM is a more selective process, providing a higher degree of confidence. Nevertheless, because this approach has not yet been validated using a standard reference material (underway), these results for tocopherols are preliminary results.

Skepticism was also applied to observation of the oxo-FA, oxo-DAGs, and oxo-TAGs. To prove that these were not produced by oxidation during the Folch extraction process, we conducted experiments in which all solvents were deaerated with argon, and extractions were done using both cold solvents and room temperature solvents. The oxo-DAGs and oxo-TAGs were present in all samples regardless of treatment. Thus, we can conclude that these did contain uncommon oxo-FAs. We doubt that these are hydroxyl-FAs because they lack the very common dehydration products, $-\mathrm{H}_{2} \mathrm{O}=\Delta 18 \mathrm{Da}$, that appear with large abundances in ESI-MS/MS and APCI-MS spectra of hydroxyl-TAGs. $^{41,42}$

It should be pointed out that the ${ }^{2} \mathrm{D}$ solvent system $(\mathrm{MeOH} /$ $\mathrm{ACN})$ is entirely compatible and miscible with the ${ }^{1} \mathrm{D}$ solvent system $(\mathrm{MeOH} / \mathrm{EtOH} / \mathrm{DCM})$, thereby eliminating all compatibility issues that can arise using hexane-based solvent systems for Ag-ion HPLC. Also, the column-switching valve was plumbed in countercurrent mode (first in, last out) because the polyunsaturated TAGs that eluted first in the ${ }^{1} \mathrm{D}$ eluted last in the ${ }^{2} \mathrm{D}$. Furthermore, cis-polyunsaturated DAGs and TAGs did not elute from the Ag-ion UHPLC column until the ACN content reached a sufficient level, so there was a degree of sample reconcentration at the head of the column, contributing to the sharp peaks seen in Figures 7 and 8 .

When referring to the 1 and 3 positions of the glycerol backbone, we no longer use the designation $s n$ (stereospecific numbering), because NARP HPLC, Ag-ion UHPLC, ESI-MS, APCI-MS, and APPI-MS are not capable of distinguishing enantiomers. The only position that can be known from these data is the $s n-2$ position. Chiral chromatography is required to differentiate the $s n-1$ and $s n-3$ positions. Therefore, the labels for the 1 and 3 positions are interchangeable unless chiral chromatography has been applied. 


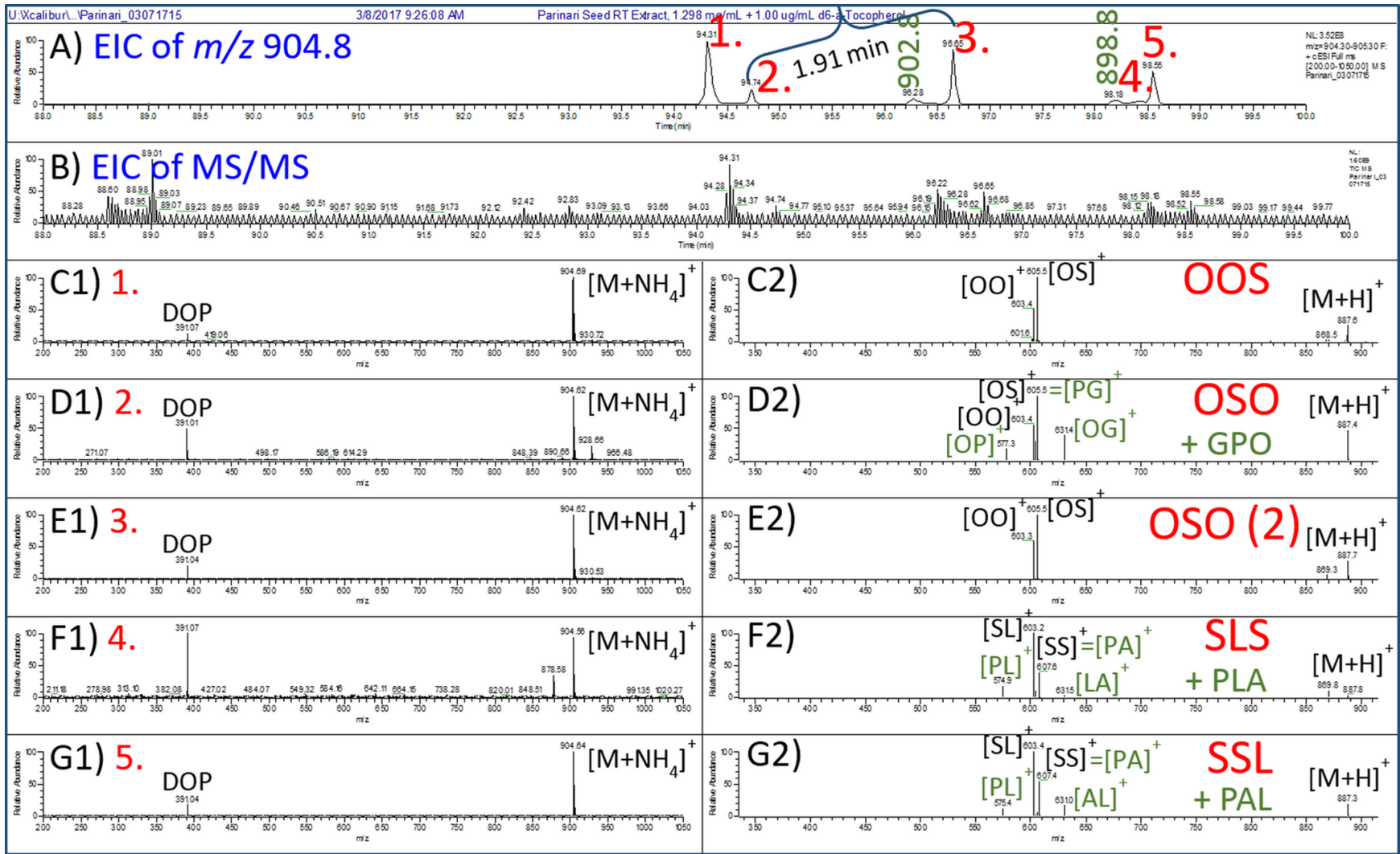

Figure 9. Extracted ion chromatograms and mass spectra of $\mathrm{m} / \mathrm{z} 904.8$ by Ag-ion UHPLC ESI-MS and MS/MS showing differentiation of regioisomers. DOP: dioctyl phthalate (plasticizer). FA abbreviations are in the Experimental Section.

An interesting observation that was unique to PSO was the difference in the ratios of the intact DAG peaks shown in Table 3 (in the column labeled DAG pk. 2/1). In most seed oils, the $1,2+2,3-\mathrm{DAG}$ peak elutes earlier and is $\sim 2$ times larger than the 1,3-DAG peak, which is smaller and elutes just after the larger peak. In PSO, even normal DAGs like LL showed a larger second peak as well as El-containing DAGs like ElEl and ElL and oxo-DAGs, oxo-ElEl, oxo-ElL, etc.

Another interesting observation was that APCI-MS and MS/ MS spectra of di-El containing TAGs (ElElEl, ElElL, ElElO, etc.) showed almost exclusively the $[\mathrm{ElEl}]^{+}[\mathrm{DAG}]^{+}, m / z 595.5$, with very little of the $[\mathrm{ElX}]^{+}[\mathrm{DAG}]^{+}$fragment. This unique behavior may indicate the possibility of cross-linking of the diEl FA chains during ionization in the APCI source, making it energetically unfavorable for formation of the $[\mathrm{ElX}]^{+}$fragment. This possibility was also indicated by the appearance of more of a $m / z 593$ fragment in ESI-MS/MS and APCI-MS mass spectra (Figures 4 and 5) than is formed from normal LnLnLn, which forms virtually only the expected $\mathrm{m} / z$ 595.5. Furthermore, TAGs containing oxElEl behaved in a similar manner, producing $m / z 609.5$ with little or no $[\mathrm{ElL}]^{+},[\mathrm{ElO}]^{+}$, or other related $[\mathrm{DAG}]^{+}$fragments, as in Figure 4.

Finally, some readers may believe that this system of two chromatographs with four mass spectrometers is prohibitively complex or expensive and cannot readily be replicated. We want to point out that researchers may take aspects of the experiments that are needed and leave unnecessary parts behind. If we had only one mass spectrometer for the ${ }^{1} \mathrm{D}$, we would use the HRAM Q Exactive Orbitrap instrument in APCI-MS mode. This would still allow identification of unknowns by HRAM MS, while also allowing quantification of FSVs, most of which do not respond well to ESI-MS without derivatization. Some may not be interested in 2D-LC at all, but the demonstration of Ag-ion UHPLC can be applied to standalone UHPLC in new ways. This arrangement of experiments was not expensive, because the slow ${ }^{2} \mathrm{D}$ chromatography and maintaining and repairing instruments ourselves allows us to keep older, inexpensive instruments in service, providing valuable data long after they have been retired elsewhere. The WCCCS system was not at all expensive, makes switching between instruments in experiments very easy, and would be a valuable addition to any LC-MS lab. Thus, while we demonstrated an unprecedented series of experiments that employ a novel arrangement of instruments, many of the components and concepts can be taken and applied individually to address a wide variety of analytical problems.

\section{CONCLUSIONS}

This work represents the first report of an application employing comprehensive 2D-LC with quadruple parallel mass spectrometry, or LC1MS2 $\times$ LC1MS2, for an LC2MS4 approach. Also reported here are the first examples of production of a silver-ion UHPLC column for triacylglycerol analysis, Ag-ion UHPLC, and Ag-ion UHPLC used as the ${ }^{2} \mathrm{D}$ in comprehensive 2D-LC. This work provides the first description of intact DAGs and TAGs from P. curatellifolia seed oil, of an oxo-FA in $P$. curatellifolia along with its confirmation using HRAM ESI-MS and GC-MS, and the first quantification of oxoDAGs and oxo-TAGs for PSO. APCI-MS data provided indications of unique ionization and fragmentation mechanisms occurring in the APCI source for TAGs containing conjugated trans double bonds. This work provides the first quantification 
of tocopherols in P. curatellifolia and shows the benefit of an extract-and-shoot approach for tocopherols in cherry pit oil. Further, these experiments describe the use of slow comprehensive $2 \mathrm{D}-\mathrm{LC}$, in which the problem of undersampling is bypassed by direct detection using six detectors in the ${ }^{1} \mathrm{D}$. This allowed more flexibility in ${ }^{2} \mathrm{D}$ method development and instruments to be used that were older than those in conventional fast 2D-LC. The Ag-ion UHPLC column was ideal for separation of TAGs by type of double bond (cis versus trans) and of regioisomers based on the locations of unsaturated FAs, either in the 1,3 positions or the $s n-2$ position. These experiments employed a unique wireless communication contact closure system to coordinate all instruments.

\section{ASSOCIATED CONTENT}

\section{S Supporting Information}

The Supporting Information is available free of charge on the ACS Publications website at DOI: 10.1021/acs.analchem. $7 \mathrm{~b} 02753$.

Experimental details for the Folch extraction, FAME preparation, GC-FID, and GC-MS analysis conditions, HPLC and UHPLC systems, and all mass spectrometers; external standard results for tocopherols by APCI-MS and internal standard and external standard results by UV detection; detailed compositions of FAMEs, DAGs, and TAGs for PSO, CPO, and SBO; and ${ }^{2} \mathrm{D}$ UHPLC contour plots for $\mathrm{CPO}$ and $\mathrm{SBO}$ (PDF)

\section{AUTHOR INFORMATION}

\section{Corresponding Author}

*E-mail: C.Byrdwell@ars.usda.gov.

\section{ORCID $\odot$}

William C. Byrdwell: 0000-0001-8241-428X

Notes

The author declares no competing financial interest.

\section{ACKNOWLEDGMENTS}

The work of Dr. Robert Goldschmidt to extract the oil and perform the analysis of fatty acid methyl esters by GC-FID and confirmation of identities by GC-MS is gratefully acknowledged. The work of Mr. Lucas Stolp and Dr. Dharma Kodali to provide the CPO sample is gratefully acknowledged. This work was supported by the USDA Agricultural Research Service. Mention or use of specific products or brands does not represent or imply endorsement by the USDA.

\section{REFERENCES}

(1) Dugo, P.; Cacciola, F.; Kumm, T.; Dugo, G.; Mondello, L. J. Chromatogr. A 2008, 1184, 353-368.

(2) Malerod, H.; Lundanes, E.; Greibrokk, T. Anal. Methods 2010, 2, $110-122$.

(3) Bedani, F.; Schoenmakers, P. J.; Janssen, H. G. J. Sep. Sci. 2012, 35, 1697-1711.

(4) Donato, P.; Cacciola, F.; Tranchida, P. Q.; Dugo, P.; Mondello, L. Mass Spectrom. Rev. 2012, 31, 523-559.

(5) Cacciola, F.; Donato, P.; Mondello, L.; Dugo, P. In Handbook of Advanced Chromatography/Mass Spectrometry Techniques; Holčapek, M., Byrdwell, W. C., Eds.; Elsevier/AOCS Press: Champaign, IL, 2017; pp 350-450.

(6) Stoll, D. R. In Handbook of Advanced Chromatography/Mass Spectrometry Techniques; Holčapek, M., Byrdwell, W. C., Eds.; Elsevier/ AOCS Press: Champaign, IL, 2017; pp 250-350.
(7) Marriott, P. J.; Schoenmakers, P.; Wu, Z. Y. LC-GC Eur. 2012, 25,

(8) Schoenmakers, P.; Marriott, P.; Beens, J. LC-GC Eur. 2003, 16, 335-339.

(9) Davis, J. M.; Stoll, D. R.; Carr, P. W. Anal. Chem. 2008, 80, 461473.

(10) Mondello, L.; Tranchida, P. Q.; Stanek, V.; Jandera, P.; Dugo, G.; Dugo, P. J. Chromatogr. A 2005, 1086, 91-98.

(11) Dugo, P.; Kumm, T.; Crupi, M. L.; Cotroneo, A.; Mondello, L. J. Chromatogr. A 2006, 1112, 269-275.

(12) Dugo, P.; Kumm, T.; Chiofalo, B.; Cotroneo, A.; Mondello, L. J. Sep. Sci. 2006, 29, 1146-1154.

(13) van der Klift, E. J. C.; Vivó-Truyols, G.; Claassen, F. W.; van Holthoon, F. L.; van Beek, T. A. J. Chromatogr. A 2008, 1178, 43-55.

(14) Yang, Q.; Shi, X.; Gu, Q.; Zhao, S.; Shan, Y.; Xu, G. J. Chromatogr. B: Anal. Technol. Biomed. Life Sci. 2012, 895-896, 48-55.

(15) Wei, F.; Ji, S. X.; Hu, N.; Lv, X.; Dong, X. Y.; Feng, Y. Q.; Chen, H. J. Chromatogr. A 2013, 1312, 69-79.

(16) Hu, J.; Wei, F.; Dong, X. Y.; Lv, X.; Jiang, M. L.; Li, G. M.; Chen, H. J. Sep. Sci. 2013, 36, 288-300.

(17) Bang, D. Y.; Moon, M. H. J. Chromatogr. A 2013, 1310, 82-90.

(18) Holčapek, M.; Ovčačíková, M.; Lísa, M.; Cífková, E.; Hájek, T. Anal. Bioanal. Chem. 2015, 407, 5033-5043.

(19) Byrdwell, W. C. Anal. Bioanal. Chem. 2011, 401, 3317-3334.

(20) Christie, W. W. J. High Resolut. Chromatogr. 1987, 10, 148-150.

(21) Ogungbenle, H. N.; A, A. A. Br. Biotechnol. J. 2014, 4, 379-386.

(22) Abolaji, O. A.; Adebayo, A. H.; Odesanmi, O. S. Pak. J. Nutr. 2007, 6, 665-668.

(23) Chisholm, M. J.; Hopkins, C. Y. J. Am. Oil Chem. Soc. 1966, 43, 390-392.

(24) Cornelius, J. A.; Hammonds, T. W.; Leicester, J. B.; Ndabahweji, J. K.; Rosie, D. A.; Shone, G. G. J. Sci. Food Agric. 1970, 21, 49-50.

(25) Sommerfeld, M. Prog. Lipid Res. 1983, 22, 221-233.

(26) Byrdwell, W. C. J. Lab. Autom. 2014, 19, 461-467.

(27) Korlesky, N. M.; Stolp, L. J.; Kodali, D. R.; Goldschmidt, R.; Byrdwell, W. C. J. Am. Oil Chem. Soc. 2016, 93, 1-11.

(28) Byrdwell, W. C.; Neff, W. E.; List, G. R. J. Agric. Food Chem. 2001, 49, 446-457.

(29) Byrdwell, W. C.; Emken, E. A.; Neff, W. E.; Adlof, R. O. Lipids 1996, 31, 919-935.

(30) Byrdwell, W. C. J. Chromatogr. A 2013, 1320, 48-65.

(31) Ball, G. F. M. Fat-Soluble Vitamin Assays in Food Analysis: A Comprehensive Review; Elsevier Science Publishing: New York, 1988.

(32) Matos, J. T. V.; Duarte, R. M. B. O.; Duarte, A. C. J. Chromatogr. B: Anal. Technol. Biomed. Life Sci. 2012, 910, 31-45.

(33) Place, B. J.; Morris, M. J.; Phillips, M. M.; Sander, L. C.; Rimmer, C. A. J. Chromatogr. A 2014, 1368, 107-115.

(34) Cook, D. W.; Rutan, S. C.; Stoll, D. R.; Carr, P. W. Anal. Chim. Acta 2015, 859, 87-95.

(35) Donato, P.; Rigano, F.; Cacciola, F.; Schure, M.; Farnetti, S.; Russo, M.; Dugo, P.; Mondello, L. J. Chromatogr. A 2016, 1458, 5462.

(36) Morris, L. J. J. Lipid Res. 1966, 7, 717-732.

(37) Byrdwell, W. C. Anal. Bioanal. Chem. 2015, 407, 5143-5160.

(38) Byrdwell, W. C. J. Am. Oil Chem. Soc. 2015, 92, 1533-1547.

(39) Fauconnot, L.; Hau, J.; Aeschlimann, J. M.; Fay, L. B.; Dionisi, F. Rapid Commun. Mass Spectrom. 2004, 18, 218-224.

(40) Powell, W. S. Anal. Biochem. 1981, 115, 267-277.

(41) Byrdwell, W. C.; Neff, W. E. J. Liq. Chromatogr. Relat. Technol. 1998, 21, 1485-1501.

(42) Byrdwell, W. C.; Neff, W. E. Rapid Commun. Mass Spectrom. 2002, 16, 300-319. 


\title{
Supporting Information for
}

\section{Comprehensive Dual Liquid Chromatography with Quadruple Mass Spectrometry (LC1MS2×LC1MS2} = LC2MS4) for Analysis of Parinari Curatellifolia and Other Seed Oil Triacylglycerols

\author{
William C. Byrdwell*
}

U.S.D.A., Agricultural Research Service, Beltsville Human Nutrition Research Center, Food Composition and Methods Development Lab, 10300 Baltimore Ave., Bldg. 161, Beltsville, MD 20705.

\section{Contents of Supporting Information:}

1. Experimental details for the Folch extraction.

- Chemicals

- Qualitative standards

- Folch extraction of Parinari curatellifolia seeds

2. Experimental details for FAME preparation, GC-FID, and GC-MS analysis conditions.

- GC apparatus

- GC-FID method

- GC-MSD method

3. Experimental details for HPLC and UHPLC systems.

- Agilent 1200 HPLC

$0 \quad{ }^{1} \mathrm{D}$ gradient for elution of fat-soluble vitamins (FSV) (Table S-1)

o $\quad{ }^{1} D$ gradient for elution of seed oil extracts (Table S-2)

$0 \quad{ }^{1} \mathrm{D}$ detector settings (Table $\mathrm{S}-3$ )

- UV diode array detector (DAD) settings

- Fluorescence detector (FLD) settings

- Charged aerosol detector (CAD) settings

- Evaporative light scattering detector (ELSD) settings

- $\quad$ Agilent 1290 UHPLC

$0 \quad{ }^{2} D$ shifted gradient for elution of FSV (Table S-4 and Figure S- 1 )

$0 \quad{ }^{2} \mathrm{D}$ shifted gradient for elution of seed oil extracts

- Cherry pit oil (CPO) and parinari seed oil (PSO) (Table S-5 and Fig. S-2)

- Wild soybean oil (SBO) (Glycine soja) (Table S-6 and Figure S-3)

$0 \quad{ }^{2} \mathrm{D}$ detector settings (Table $\mathrm{S}-7$ )

- UV diode array detector settings

4. Experimental details for all mass spectrometers: HRAM ESI-MS on QExactive, APCI-MS on TSQ Vantage EMR, APPI-MS on TSQ Quantum Access Max, and ESI-MS on LCQ Deca XP.

- $\quad{ }^{1} D$ QExactive high-resolution accurate-mass (HRAM) instrument methods (FSV and oils) (Table S-8)

- $\quad{ }^{1} D$ TSQ Vantage EMR instrument method for FSV (Table S-9)

- $\quad{ }^{1}$ D TSQ Vantage EMR instrument method for CPO and PSO (Table S-10)

- $\quad{ }^{2} D$ TSQ Quantum Access Max instrument methods (FSV and oils) (Table S-11)

- ${ }^{2}$ D LCQ Deca XP instrument methods (FSV and oils) (Table S-12)

5. External standard results for tocopherols by APCI-MS, internal standard and external standard results by UV detection.

- Internal standard (IS) and external standard (ES) results by UV and ES results by APCI-MS (Table S-13)

- Limits of detection (LOD) and limits of quantification (LOQ) from calibration lines (Table S-14)

6. Detailed response factor normalized (RFN) and GC-FID compositions of FAMEs for PSO, CPO, and SBO.

- RFN fatty acid composition of PSO, with compositions of 18:3 and oxo-El isomers (Table S-15)

- RFN fatty acid composition of CPO (Table S-16)

- RFN fatty acid composition of SBO (Table S-17)

7. Detailed compositions of DAGs for CPO, PSO, and SBO.

- RFN Diacylglycerol (DAG) composition of CPO by APCI-MS (Table S-18) 
- More detailed RFN DAG composition of Parinari curatellifolia seed oil extract (Table S-19)

- RFN DAG composition of SBO by APCI-MS (Table S-20)

8. Detailed compositions of TAGs for CPO, PSO, and SBO.

- Detailed RFN triacylglycerol (TAG) composition of CPO by APCI-MS (Table S-21)

- Detailed RFN TAG composition of PSO by APCI-MS (Table S-22)

- Detailed RFN TAG composition of SBO by APCI-MS (Table S-23)

9. ${ }^{2} \mathrm{D}$ UHPLC contour plot for CPO.

- $\quad{ }^{2}$ D UHPLC Plots for CPO by APPI-MS on TSQ Quantum Access Max mass spectrometer (Fig. S-4)

- $\quad{ }^{2}$ D UHPLC Plots for CPO by ESI-MS on LCQ Deca XP mass spectrometer (Fig. S-5)

10. ${ }^{2} \mathrm{D}$ UPHLC contour plot for SBO.

- $\quad{ }^{2}$ D UHPLC Plots for SBO by APPI-MS on TSQ Quantum Access Max mass spectrometer (Fig. S-6)

- $\quad{ }^{2}$ D UHPLC Plots for SBO by ESI-MS on LCQ Deca XP mass spectrometer (Fig. S-7)

\section{Experimental Section}

Chemicals

a) Boron Trifluoride $\left(\mathrm{BF}_{3}\right)-14 \%$ in methanol obtained from Sigma-Aldrich (St. Louis, $\mathrm{MO}$ ).

b) Chloroform - Sigma-Aldrich $99.9 \%$ A.C.S. HPLC grade.

c) Dichloromethane (DCM) - Fisher Scientific (Pittsburgh, PA) Optima grade.

d) Ethanol (EtOH) - Sigma-Aldrich $100 \%$ (200 proof) HPLC/Spectrophotometric grade.

e) Isooctane (2,2,4-trimethyl pentane) - Mallinckrodt (Paris, KY).

f) Methanol $(\mathrm{MeOH})$ - Fisher Scientific Optima LC/MS grade.

g) Potassium chloride $(\mathrm{KCl})$ - certified A.C.S. grade obtained from Fisher Scientific.

h) Sodium chloride ( $\mathrm{NaCl}$ - A.C.S. reagent grade obtained from Sigma (St. Louis, $\mathrm{MO}$ ).

i) Sodium Hydroxide ( $\mathrm{NaOH})$ - A.C.S. reagent grade obtained from Aldrich (Milwaukee, WI).

j) Water - Obtained from in-house Millipore (Bedford, MA) Milli-Q Water Purification System.

\section{Qualitative Standards}

a) Multi-component FAME Standard - GLC Reference Standard 68B from Nu-Check Prep (Elysian, MN) contains 18 FAME derivatives of fatty acids ranging from C14 to C24 and was prepared as a solution at $100 \mathrm{mg}$ in $50 \mathrm{~mL}$ isooctane.

b) Multi-component FAME Standard - GLC Reference Standard 14B from Nu-Check Prep contains the FAME derivatives of the five odd carbon number unsaturated fatty acids from C13:0 to C21:0. It was prepared as a solution at $100 \mathrm{mg}$ in $50 \mathrm{~mL}$ isooctane.

c) Methyl Tricosanoate (FAME derivative of C23:0 fatty acid) - Obtained from Nu-Check Prep and prepared as a solution at $15 \mathrm{mg}$ in $25 \mathrm{~mL}$ isooctane.

d) Methyl Pentacosanoate (FAME derivative of C25:0 fatty acid) - Obtained from Santa Cruz Biotechnology, Inc. (Dallas, TX) and prepared as a solution at $15 \mathrm{mg}$ in $25 \mathrm{~mL}$ isooctane.

e) Methyl hexacosanoate (FAME derivative of C26:0 fatty acid) - Obtained from Matreya LLC (Pleasant Gap, PA) and prepared as a solution at $15 \mathrm{mg}$ in $25 \mathrm{~mL}$ isooctane.

\section{Folch Extraction of Parinari curatellifolia Seeds}

Parinari curatellifolia seeds were obtained from Top Tropicals (Ft. Myers, FL). Seeds were placed in a $-80{ }^{\circ} \mathrm{C}$ freezer for two hours. The hard, thick shells were then cracked with a hammer, and the inner, white endosperms were obtained. The average seed mass, including the shell, was $5.2 \mathrm{~g}$, and the average recovered portion was $0.41 \mathrm{~g}$, or about $8 \%$ of the total. The endosperms were ground in an electric grinder. The resulting oily meal was collected, and $0.2 \mathrm{~g}$ portions were weighed for extraction. The extraction of Folch et al. ${ }^{1}$ was performed using two successive $4 \mathrm{~mL}$ portions of 2:1 chloroform: methanol. For the initial portion stirring was for $20 \mathrm{~min}$. Samples were then centrifuged at $1000 \mathrm{rpm}$ for $2 \mathrm{~min}$ and the supernatant was recovered. This procedure was then repeated, but for the second portion stirring was for $5 \mathrm{~min}$. The recovered extracts were 
combined and washed with $2 \mathrm{~mL}$ of aqueous $0.1 \% \mathrm{KCl}$. A final centrifugation at $1000 \mathrm{rpm}$ for 2 min was performed, and the lower, chloroform layers were collected. Solvent evaporation to constant mass was performed at room temperature under a stream of nitrogen gas. The final, oily residues obtained were on average $45 \%$ by mass of the ground portions weighed for extraction. A solution of $67.6 \mathrm{mg}$ PSO in $50 \mathrm{~mL}$ deaerated (da-) dichloromethane (DCM) / methanol (MeOH) $(1: 1),=1.352 \mathrm{mg} / \mathrm{mL}$, was initially prepared and kept at $-2{ }^{\circ} \mathrm{C}$. Later, this solution was brought to room temperature and $25.0 \mathrm{~mL}$ was transferred to a $25.0 \mathrm{~mL}$ flask, $1.0 \mathrm{~mL}$ was removed, and $1.0 \mathrm{~mL}$ of $25.0 \mu \mathrm{g} / \mathrm{mL} \mathrm{d} \mathrm{d}_{6}$ - $\alpha$-tocopherol internal standard (IS) was added to give $1.298 \mathrm{mg} / \mathrm{mL}$ P. curatellifolia with $1.0 \mu \mathrm{g} / \mathrm{mL}$ IS.

The CPO was obtained by hexane Soxhlet extraction as previously described. ${ }^{2} 1.3768 \mathrm{mg}$ was added to an amber volumetric flask, $\sim 30 \mathrm{~mL}$ of da-DCM/MeOH (1:1) was added with swirling, $4.0 \mathrm{~mL}$ of $25.0 \mu \mathrm{g} / \mathrm{mL} \mathrm{d} \mathrm{d}_{6}-\alpha-$ tocopherol IS was added, and the solution was made to $100 \mathrm{~mL}$ with da-DCM/MeOH (1:1) to give 1.3768 $\mathrm{mg} / \mathrm{mL}$ oil with $1.0 \mu \mathrm{g} / \mathrm{mL} \mathrm{IS}$.

An 8 fluid ounce bottle of organic wild soybean (Glycine soja) oil (SBO) with an expiration date of 11/2019 was purchased from an online marketplace and was used as received. A solution of $1.3162 \mathrm{mg} / \mathrm{mL} \mathrm{SBO}$ with 1.0 $\mu \mathrm{g} / \mathrm{mL} \mathrm{d}_{6}$ - $\alpha$-tocopherol IS was prepared the same way as the CPO.

\section{FAME Preparation of Oil Samples and GC Analysis}

For all oil samples FAME derivatives were prepared as described in AOCS Official Method Ce $\mathbf{1 b}-\mathbf{8 9}$ with the following modifications:

a) The parts of the method related to the absolute determination of eicosapentaenoic acid (EPA) and docosahexaenoic acid (DHA) were omitted.

b) The entire procedure was performed at room temperature $\left(22^{\circ} \mathrm{C}\right)$. Saponification with $0.5 \mathrm{M}$ methanolic $\mathrm{NaOH}$ was overnight (16-20 hrs), and $\mathrm{BF}_{3}$ treatment was for $45-60$ min.

c) For each sample, two extractions using $1 \mathrm{~mL}$ isooctane for each were performed; however, the evaporation of the combined extracts to $1 \mathrm{~mL}$ was omitted.

\section{GC Apparatus}

a) GC column - Supelco (Bellefonte, PA) Omegawax 250, $30 \mathrm{~m} \times 0.25 \mathrm{~mm} \times 0.25 \mu \mathrm{m}$ film thickness.

b) Gas Chromatograph - Agilent Technologies (Santa Clara, CA) 6890N GC with G2613A injector, split/splitless inlet, flame ionization detector (FID), and using OpenLab CDS ChemStation Edition for GC Systems (Rev. C.01.05 [35]) software.

c) GC-MS - Agilent Technologies 5975C Inert XL EI/CI MSD with 7890A GC System and G4513A injector, split/splitless inlet, and using MassHunter GC/MS Acquisition (B.07.00 SP1.1549) software.

\section{GC-FID Method}

The GC-FID method was as described in AOCS Official Method Ce $\mathbf{1 b - 8 9}$ with the following specifications and modifications:

a) Carrier gas - Helium with operation in constant flow mode at $1 \mathrm{~mL} / \mathrm{min}$.

b) FID - Temperature set at $270{ }^{\circ} \mathrm{C}$ with hydrogen flow at $45 \mathrm{~mL} / \mathrm{min}$, air flow at $450 \mathrm{~mL} / \mathrm{min}$, and makeup gas (Nitrogen) flow at $45 \mathrm{~mL} / \mathrm{min}$.

c) Inlet - Temperature set at $250^{\circ} \mathrm{C} ; 1 \mu \mathrm{L}$ injection; operation in split mode at 50:1 split.

d) Oven - Initial temperature of $170^{\circ} \mathrm{C}$, temperature gradient of $1{ }^{\circ} \mathrm{C} / \mathrm{min}$ to $250^{\circ} \mathrm{C}$.

e) Run Time $-80 \mathrm{~min}$.

\section{GC-MSD Method}

The GC part of the GC-MSD method was similar to that described above for the GC-FID method. Total ion current chromatograms were acquired in scanning mode from $\mathrm{m} / \mathrm{z} 50$ to $\mathrm{m} / \mathrm{z} 450$ at 1.9 scans/second and a step size of $0.1 \mathrm{~m} / \mathrm{z}$. Samples were run using electron ionization (EI) mode and again using positive ion chemical ionization $(\mathrm{Cl})$ mode with methane reagent gas. 


\section{HPLC and MS Instruments and Conditions}

A) Agilent 1200 HPLC (Agilent Technologies, Santa Clara, CA)

Solvent module with membrane degasser (G1379B), quaternary pump (G1311A), autosampler (G1329A) with 1290 thermostat (G1330B) at $15^{\circ} \mathrm{C}$, thermostatted column compartment (G1316A), diode array detector (DAD) SL (G1315C), two-channel 24-bit analog-to-digital converter \#1 (ADC) (35900E).

Two Inertsil ODS-2 columns in series, $25 \mathrm{~cm} \times 4.6 \mathrm{~mm}, 5 \mu \mathrm{m}$ particles (GL Sciences, Torrance, CA, USA) joined by a circularly bent $7-\mathrm{cm}$ piece of 0.007 in. i.d. stainless steel tubing.

Columns were maintained at $10^{\circ} \mathrm{C}$ throughout. $20 \mu \mathrm{L}$ of standards and samples injected.

I. ${ }^{1} \mathrm{D}$ Gradient elution as follows:

1) Fat-soluble vitamin (FSV) standards:

\begin{tabular}{|c|c|c|c|c|}
\hline \multicolumn{6}{|c|}{ Table S-1. ${ }^{1}$ D HPLC gradient for fat-soluble vitamins. } \\
\hline Time (min) & \%A (MeOH) & \%B (ACN) & \%C (EtOH) & \%D (DCM) \\
\hline $\mathbf{0 . 0}$ & 95.0 & 0.0 & 5.0 & 0.0 \\
\hline $\mathbf{2 5 . 0}$ & 95.0 & 0.0 & 5.0 & 0.0 \\
\hline $\mathbf{3 0 . 0}$ & 60.0 & 0.0 & 15.0 & 25.0 \\
\hline $\mathbf{4 0 . 0}$ & 65.0 & 0.0 & 10.0 & 25.0 \\
\hline $\mathbf{5 0 . 0}$ & 65.0 & 0.0 & 10.0 & 25.0 \\
\hline $\mathbf{5 1 . 0}$ & 95.0 & 0.0 & 5.0 & 0.0 \\
\hline $\mathbf{5 4 . 0}$ & 95.0 & 0.0 & 5.0 & 0.0 \\
\hline
\end{tabular}

2) Seed oil extracts:

Table S-2. ${ }^{1}$ D HPLC gradient for seed oil extracts.

\begin{tabular}{|c|c|c|c|c|}
\hline Time (min) & \%A (MeOH) & \%B (ACN) & \%C (EtOH) & \%D (DCM) \\
\hline $\mathbf{0 . 0}$ & 95.0 & 0.0 & 5.0 & 0.0 \\
\hline $\mathbf{2 5 . 0}$ & 95.0 & 0.0 & 5.0 & 0.0 \\
\hline $\mathbf{3 0 . 0}$ & 60.0 & 0.0 & 15.0 & 25.0 \\
\hline $\mathbf{4 0 . 0}$ & 65.0 & 0.0 & 10.0 & 25.0 \\
\hline $\mathbf{5 0 . 0}$ & 65.0 & 0.0 & 10.0 & 25.0 \\
\hline $\mathbf{7 0 . 0}$ & 65.0 & 0.0 & 10.0 & 25.0 \\
\hline $\mathbf{9 0 . 0}$ & 50.0 & 0.0 & 25.0 & 25.0 \\
\hline $\mathbf{1 0 0 . 0}$ & 35.0 & 0.0 & 40.0 & 25.0 \\
\hline $\mathbf{1 1 0 . 0}$ & 30.0 & 0.0 & 45.0 & 25.0 \\
\hline $\mathbf{1 1 8 . 0}$ & 30.0 & 0.0 & 45.0 & 25.0 \\
\hline $\mathbf{1 2 0 . 0}$ & 95.0 & 0.0 & 5.0 & 0.0 \\
\hline $\mathbf{1 3 0 . 0}$ & 95.0 & 0.0 & 5.0 & 0.0 \\
\hline
\end{tabular}

II. ${ }^{1} D$ Detector settings:

Table S-3. ${ }^{1} \mathrm{D}$ HPLC detector settings.

\begin{tabular}{|c|c|c|c|c|}
\hline \multicolumn{5}{|c|}{${ }^{1}$ D Diode Array Detector (DAD) } \\
\hline Wavelength $\left(\lambda_{\mathrm{nm}}\right)$ & Bandwidth $\left(\Delta \boldsymbol{\lambda}_{\mathrm{nm}}\right)$ & Reference $\boldsymbol{\lambda}_{\mathrm{nm}}$ & Reference $\Delta \boldsymbol{\lambda}_{\mathrm{nm}}$ & Analyte(s) \\
\hline 210 & 5 & 360 & 100 & Generic \\
\hline 248 & 9 & 360 & 100 & Phylloquinone (Vit. $\left.\mathrm{K}_{1}\right)$ \\
\hline 265 & 9 & 360 & 100 & Vitamin $\mathrm{D}_{2} \& \mathrm{D}_{3}$ \\
\hline 297 & 11 & 450 & 100 & Tocopherols (Vit. E) \\
\hline 326 & 11 & 450 & 100 & $\begin{array}{c}\text { Retinol (Vit. A), Retinyl } \\
\text { Acetate, Retinyl } \\
\text { Palmitate }\end{array}$ \\
\hline
\end{tabular}




\begin{tabular}{|c|c|c|}
\hline \multicolumn{3}{|c|}{${ }^{1}$ D Fluorescence Detector (FLD) } \\
\hline $\begin{array}{c}\text { Excitation } \\
\text { Wavelength } \\
\left(\lambda_{\mathrm{nm}}\right) \\
\end{array}$ & $\begin{array}{c}\text { Emission } \\
\text { Wavelength } \\
\left(\lambda_{\mathrm{nm}}\right) \\
\end{array}$ & Analyte(s) \\
\hline Zero order [Xe lamp: $200-1200 \mathrm{~nm}$ ] & 310 & $\alpha$-Tocopheryl Acetate \\
\hline Zero order (all $\lambda$ ) & 330 & Tocopherols (Vit. E) \\
\hline Zero order (all $\lambda$ ) & 420 & $\begin{array}{c}\text { Fluorescent 18:4- } \\
\text { containing TAG reported in } \\
\text { Parinari glaberrimum }\end{array}$ \\
\hline Zero order (all $\lambda)$ & 470 & $\begin{array}{c}\text { Retinol (Vit. A), Retinyl } \\
\text { Acetate, Retinyl Palmitate }\end{array}$ \\
\hline \multicolumn{3}{|c|}{${ }^{1}$ D Corona Charged Aerosol Detector (CAD) } \\
\hline Parameter & & Setting \\
\hline Range & & $20 \mathrm{pA}$ \\
\hline Filter & & 3 \\
\hline Power function & & 1.00 \\
\hline Gas (Nitrogen) & & 35 psi \\
\hline Output offset & & $0 \%$ \\
\hline \multicolumn{3}{|c|}{${ }^{1}$ D Evaporative Light Scattering Detector (ELSD) - Not Reported due to temporary signal failure } \\
\hline Parameter & & Setting \\
\hline Evaporator & & $80^{\circ} \mathrm{C}$ \\
\hline Nebulizer & & $90^{\circ} \mathrm{C}$ \\
\hline Gas (Nitrogen) & & 1.20 \\
\hline Photomultiplier & & 8.4 \\
\hline Data Rate & & $40 \mathrm{~Hz}$ \\
\hline Smoothing & & 5 \\
\hline
\end{tabular}

\section{B) Agilent 1290 UHPLC (Agilent Technologies, Santa Clara, CA)}

Solvent module with membrane degasser, Infinity binary pump (G4220A), Infinity autosampler (G4226A disconnected) with FC/ALS Thermostat (G1330B), thermostatted column compartment (G1316C), Infinity diode array detector (DAD) (G4212A), 8-port, 2-position, 1200 bar column-switching valve (G1170A). Lab-made Ag-Ion column from ES Industries strong cation exchange column (Epic-SCX, \#122191-ESCX), $10.0 \mathrm{~cm}$ $\times 2.1 \mathrm{~mm}, 3 \mu \mathrm{m}, 120 \AA$, particles (ES Industries, West Berlin, NJ, USA).

Column was maintained at $10^{\circ} \mathrm{C}$ throughout. Gradient stop time $1.86 \mathrm{~min}$. Modulation time $1.91 \mathrm{~min}$.

I. ${ }^{2} \mathrm{D}$ Shifted gradient elution as follows:

1) Fat-soluble vitamin (FSV) standards:

Table S-4. ${ }^{2}$ D UHPLC shifted gradient for fat-soluble vitamins. See Figure S-1.

\begin{tabular}{|c|c|c|c|c|c|}
\hline & \multicolumn{5}{|c|}{${ }^{2} \mathrm{D}$ Time (min) } \\
\hline${ }^{1} \mathrm{D}$ Time (min) & 0.00 & 0.55 & 1.5 & 1.70 & 1.80 \\
\hline 0.0 & 100.0 & 100.0 & 90.0 & 90.0 & 100.0 \\
\hline 40.0 & 100.0 & 100.0 & 90.0 & 90.0 & 100.0 \\
\hline 50.0 & 100.0 & 100.0 & 10.0 & 10.0 & 100.0 \\
\hline 60.0 & 100.0 & 100.0 & 35.0 & 35.0 & 100.0 \\
\hline 70.0 & 100.0 & 100.0 & 60.0 & 60.0 & 100.0 \\
\hline 120.0 & 100.0 & 100.0 & 90.0 & 90.0 & 100.0 \\
\hline 130.0 & 100.0 & 100.0 & 90.0 & 90.0 & 100.0 \\
\hline
\end{tabular}


2) Cherry (Prunus cerasus) and Parinari curatellifolia seed oil extracts:

Table S-5. ${ }^{2}$ D UHPLC shifted gradient for cherry and parinari seed oil extracts. See Figure S-2.

\begin{tabular}{|c|c|c|c|c|c|}
\hline \multicolumn{5}{|c|}{${ }^{\mathbf{2}} \mathbf{D}$ Time (min) } \\
\hline${ }^{1} \mathbf{D}$ Time (min) & $\mathbf{0 . 0 0}$ & $\mathbf{0 . 5 5}$ & $\mathbf{1 . 5}$ & $\mathbf{1 . 7 0}$ & $\mathbf{1 . 8 0}$ \\
\hline $\mathbf{0 . 0}$ & 100.0 & 100.0 & 90.0 & 90.0 & 100.0 \\
\hline $\mathbf{4 0 . 0}$ & 100.0 & 100.0 & 90.0 & 90.0 & 100.0 \\
\hline $\mathbf{5 0 . 0}$ & 100.0 & 100.0 & 10.0 & 10.0 & 100.0 \\
\hline $\mathbf{6 0 . 0}$ & 100.0 & 100.0 & 35.0 & 35.0 & 100.0 \\
\hline $\mathbf{7 0 . 0}$ & 100.0 & 100.0 & 60.0 & 60.0 & 100.0 \\
\hline $\mathbf{1 2 0 . 0}$ & 100.0 & 100.0 & 90.0 & 90.0 & 100.0 \\
\hline $\mathbf{1 3 0 . 0}$ & 100.0 & 100.0 & 90.0 & 90.0 & 100.0 \\
\hline
\end{tabular}

3) Commercial wild soybean (Glycine soja) oil:

Table S-6. ${ }^{2}$ D UHPLC shifted gradient for cherry and parinari seed oil extracts. See Figure S-3.

\begin{tabular}{|c|c|c|c|c|c|}
\hline & \multicolumn{5}{|c|}{${ }^{\mathbf{2}} \mathbf{D}$ Time (min) } \\
\hline${ }^{\mathbf{1}} \mathbf{D}$ Time (min) & $\mathbf{0 . 0 0}$ & $\mathbf{0 . 5 5}$ & $\mathbf{1 . 5}$ & $\mathbf{1 . 7 0}$ & $\mathbf{1 . 8 0}$ \\
\hline $\mathbf{0 . 0}$ & 100.0 & 100.0 & 95.0 & 95.0 & 100.0 \\
\hline $\mathbf{4 0 . 0}$ & 100.0 & 100.0 & 95.0 & 95.0 & 100.0 \\
\hline $\mathbf{5 0 . 0}$ & 100.0 & 100.0 & 25.0 & 10.0 & 100.0 \\
\hline $\mathbf{6 0 . 0}$ & 100.0 & 100.0 & 40.0 & 35.0 & 100.0 \\
\hline $\mathbf{7 0 . 0}$ & 100.0 & 100.0 & 90.0 & 90.0 & 100.0 \\
\hline $\mathbf{1 2 0 . 0}$ & 100.0 & 100.0 & 95.0 & 95.0 & 100.0 \\
\hline $\mathbf{1 3 0 . 0}$ & 100.0 & 100.0 & 95.0 & 95.0 & 100.0 \\
\hline
\end{tabular}

II. ${ }^{2} D$ Detector settings:

Table S-7. ${ }^{2} \mathrm{D}$ HPLC detector settings (Identical to ${ }^{1} \mathrm{D}$ DAD settings).

${ }^{2} \mathrm{D}$ Diode Array Detector (DAD)

\begin{tabular}{|c|c|c|c|c|}
\hline Wavelength $\left(\lambda_{\mathrm{nm}}\right)$ & Bandwidth $\left(\Delta \lambda_{\mathrm{nm}}\right)$ & Reference $\lambda_{\mathrm{nm}}$ & Reference $\Delta \lambda_{\mathrm{nm}}$ & Analyte \\
\hline 210 & 5 & 360 & 100 & Generic \\
\hline 248 & 9 & 360 & 100 & Phylloquinone (Vit. $\left.\mathrm{K}_{1}\right)$ \\
\hline 265 & 9 & 360 & 100 & Vitamin $\mathrm{D}_{2} \& \mathrm{D}_{3}$ \\
\hline 297 & 11 & 450 & 100 & Tocopherols (Vit. E) \\
\hline 326 & 11 & 450 & 100 & $\begin{array}{c}\text { Retinol (Vit. A), Retinyl } \\
\text { Acetate, Retinyl } \\
\text { Palmitate }\end{array}$ \\
\hline
\end{tabular}




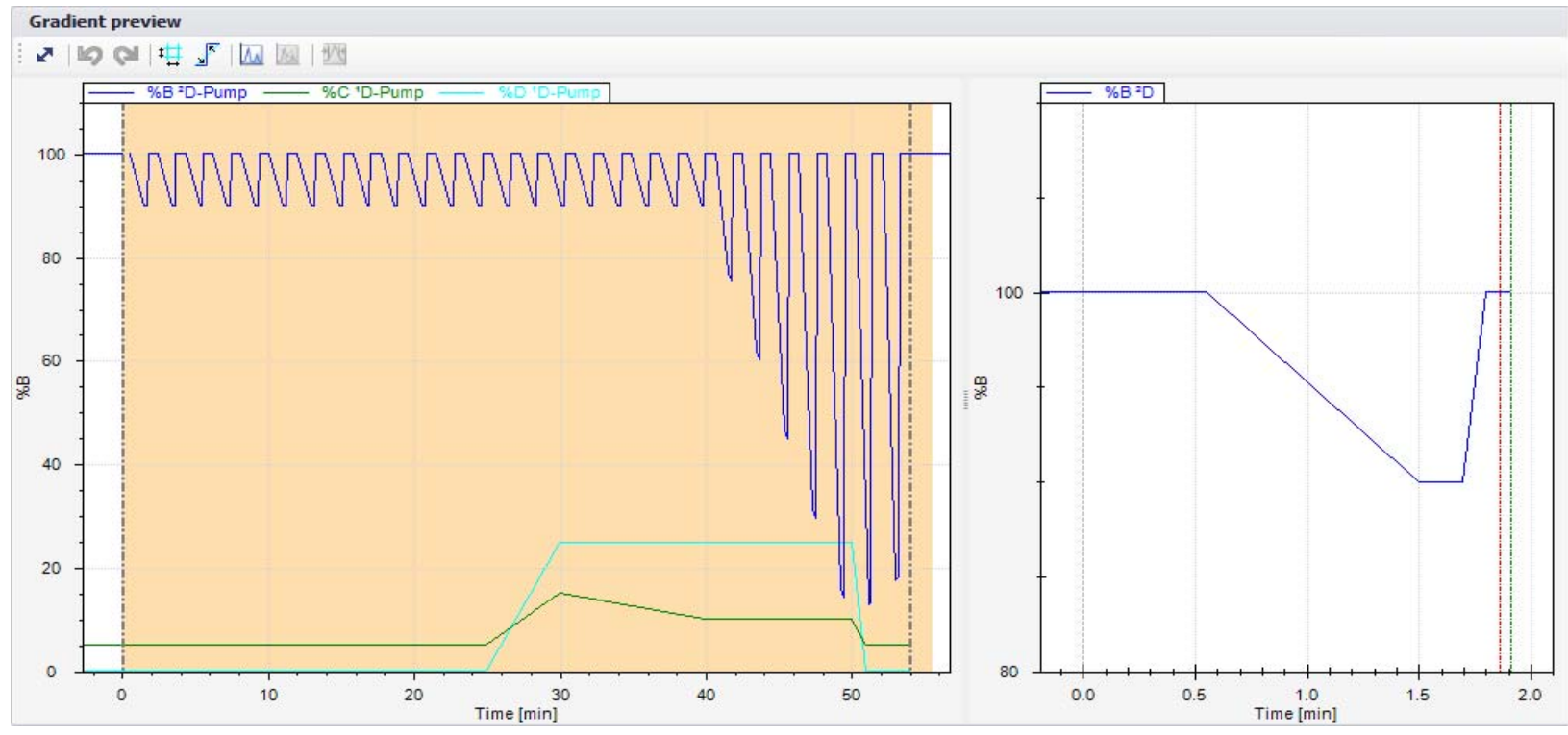

Figure S-1. ${ }^{2}$ D UHPLC shifted gradient for fat-soluble vitamins. See Table S-4.

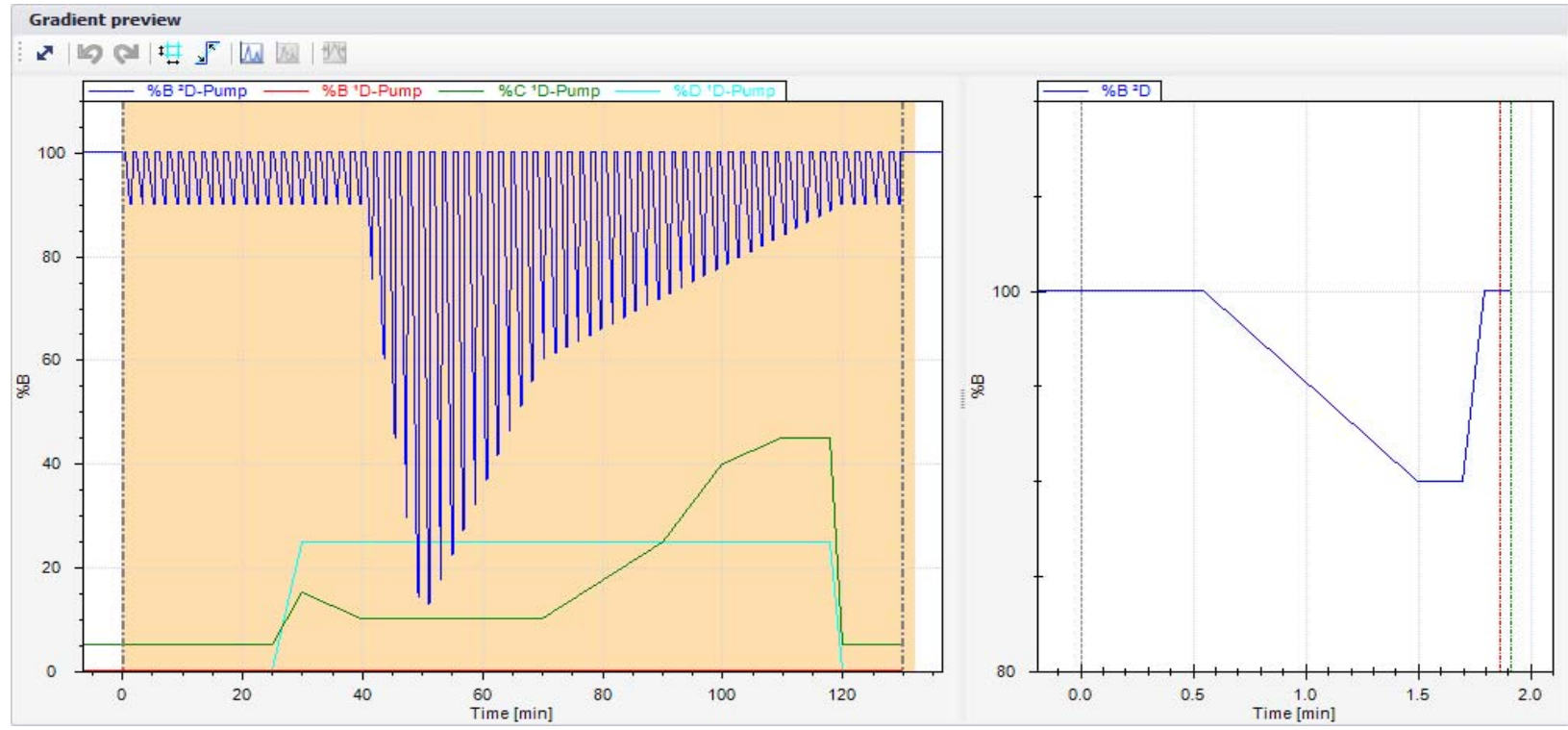

Figure S-2. ${ }^{2}$ D UHPLC shifted gradient for cherry and parinari seed oil extracts. See Table S-5.
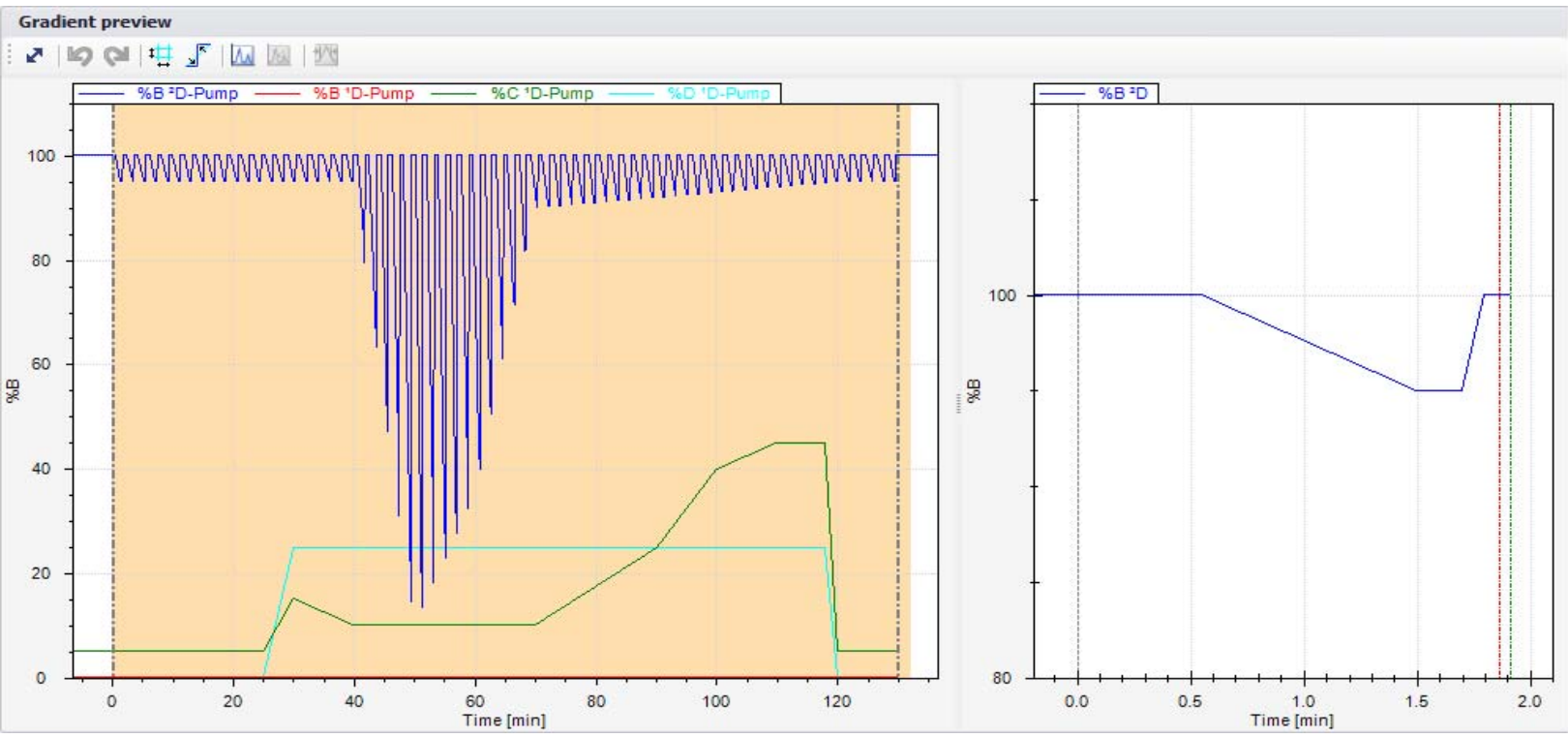

Figure S-3. ${ }^{2}$ D UHPLC shifted gradient for commercial wild soybean (Glycine soja) samples. See Table S-6. 
4. Mass Spectrometer Operating Conditions

A) ${ }^{1}$ D HPLC detection.

Table S-8. QExactive high resolution accurate mass Orbitrap ${ }^{\mathrm{TM}}$ mass spectrometer (Thermo Fisher Scientific, San Jose, CA, USA) for qualitative ${ }^{1}$ D HPLC analysis.

All runs use ammonium formate $\left(\mathrm{NH}_{4} \mathrm{OCOH}\right)$ in methanol (1:4) at $20 \mu \mathrm{L} / \mathrm{min}$ supplied by Applied Biosystems AB140C dual piston syringe pump.

\begin{tabular}{|c|c|c|c|}
\hline \multicolumn{4}{|c|}{ Fat-Soluble Vitamin Analysis } \\
\hline \multicolumn{2}{|c|}{ Full-Scan ESI-MS } & \multicolumn{2}{|c|}{ Data-Dependent Acquisition (DDA) MS/MS } \\
\hline Parameter & Setting & Parameter & Setting \\
\hline Source parameters & See below & In-source CID & $0.0 \mathrm{eV}$ \\
\hline Run time & $54 \mathrm{~min}$ & Precursor resolution & 140,000 \\
\hline In-source CID & $45.0 \mathrm{eV}$ & Product resolution & 70,000 \\
\hline Resolution & 140,000 & Precursor scan range & $\mathrm{m} / \mathrm{z} 300-750$ \\
\hline Scan range & $m / z 200-2000$ & Loop & Top 2 precursors \\
\hline AGC target & $3 e 6$ & Isolation window & $1.0 \mathrm{~m} / \mathrm{z}$ \\
\hline Max. inj. time & $200 \mathrm{~ms}$ & Norm. collision energy & 50 \\
\hline Mode & Centroid & Max. inj. time & Pre:100 ms/Prod:50 ms \\
\hline Polarity & + and - & & \\
\hline \multicolumn{4}{|c|}{ Cherry and Parinari Extract 130 min Analysis } \\
\hline \multicolumn{4}{|c|}{ Up to $44 \mathrm{~min}$ same as FSV above. Soybean Oil the same, but first segment = $50 \mathrm{~min}$. } \\
\hline \multicolumn{2}{|c|}{ Full-Scan ESI-MS } & \multicolumn{2}{|c|}{ TAG DDA MS/MS } \\
\hline Parameter & Setting & Parameter & Setting \\
\hline Sheath gas (Nitrogen) & 25 & In-source CID & $0.0 \mathrm{eV}$ \\
\hline Auxiliary gas (Nitrogen) & 0 & Resolution & Pre:140,000/Prod:70,000 \\
\hline Sweep gas & 0 & Precursor scan range & $m / z 700-1100$ \\
\hline Spray voltage & $4000 \mathrm{~V}$ & Loop & Top 2 precursors \\
\hline Capillary temp. & $250^{\circ} \mathrm{C}$ & Isolation window & $1.0 \mathrm{~m} / \mathrm{z}$ \\
\hline Run time & $130 \mathrm{~min}$ & Norm. collision energy & 15 \\
\hline In-source CID & $45.0 \mathrm{eV}$ & \multicolumn{2}{|c|}{ DAG DDA MS/MS } \\
\hline Resolution & 140,000 & In-source CID & $80.0 \mathrm{eV}$ \\
\hline Scan range & $m / z 200-2000$ & Resolution & Pre:140,000/Prod:70,000 \\
\hline AGC target & $3 e 6$ & Precursor scan range & $m / z 350-750$ \\
\hline Max. inj. time & $200 \mathrm{~ms}$ & Loop & Top 3 precursors \\
\hline Mode & Centroid & Isolation window & $1.0 \mathrm{~m} / \mathrm{z}$ \\
\hline Polarity & + and - & Norm. collision energy & 35 \\
\hline
\end{tabular}


Table S-9. TSQ Vantage EMR tandem sector quadrupole mass spectrometer (Thermo Fisher Scientific, San Jose, CA, USA). Multi-segment qualitative and SIM and SRM quantitative analysis parameters for ${ }^{1} D$ HPLC.

\begin{tabular}{|c|c|}
\hline \multicolumn{2}{|c|}{ Fat-Soluble Vitamin Analysis } \\
\hline \multicolumn{2}{|c|}{ APCI Source Parameters } \\
\hline Parameter & Setting \\
\hline Vaporizer heater & $400{ }^{\circ} \mathrm{C}$ \\
\hline Sheath gas (Nitrogen) & 50 \\
\hline Auxiliary gas (Nitrogen) & 5 \\
\hline Sweep gas & 0 \\
\hline Capillary temp. & $250^{\circ} \mathrm{C}$ \\
\hline Declustering voltage & $0 \mathrm{~V}$ \\
\hline
\end{tabular}

1.0 mTorr Argon collision induced dissociation (CID) gas turned on throughout all scans.

All scans except precursor scans used Q3. All used 0.7 FWHM peak widths unless otherwise stated. All scans were in $(+)$ ion mode with centroided masses.

\begin{tabular}{|c|c|c|c|c|c|c|c|}
\hline \multirow{2}{*}{\multicolumn{4}{|c|}{$\frac{\text { Segment } 1 \text { (0-2 } \mathrm{min})}{\text { n event } 1 . \mathrm{Q} 3 \text { Full-scan MS }}$}} & \multicolumn{4}{|c|}{ Segment 3 - Scan Event 3-SIM Continued } \\
\hline & & & & \multirow{2}{*}{\multicolumn{2}{|c|}{$\begin{array}{c}m / z \\
403.358\end{array}$}} & \multicolumn{2}{|c|}{ Analyte } \\
\hline \multicolumn{2}{|l|}{ Scan range } & \multicolumn{2}{|c|}{$m / z 200-2000$} & & & \multicolumn{2}{|c|}{$\delta$-Tocopherol } \\
\hline \multicolumn{2}{|l|}{ Scan time } & \multicolumn{2}{|r|}{$1.8 \mathrm{~s}$} & \multicolumn{2}{|l|}{416.365} & \multicolumn{2}{|c|}{$\gamma, \beta$-Tocopherol [M] } \\
\hline \multicolumn{4}{|c|}{ Scan events 2. \& 3. DDA MS/MS } & \multicolumn{2}{|l|}{417.373} & \multicolumn{2}{|c|}{$\gamma, \beta$-Tocopherol $[\mathrm{M}+\mathrm{H}]^{+}$} \\
\hline \multicolumn{2}{|c|}{ Signal threshold } & \multicolumn{2}{|r|}{$1 \mathrm{e} 4$} & \multicolumn{2}{|l|}{431.389} & \multicolumn{2}{|c|}{$\alpha$-Tocopherol } \\
\hline \multicolumn{2}{|l|}{ Scan time } & \multicolumn{2}{|r|}{$1.0 \mathrm{~s}$} & \multicolumn{2}{|l|}{437.427} & \multicolumn{2}{|c|}{$d_{6}-\alpha-$ Tocopherol } \\
\hline \multicolumn{2}{|c|}{ Collision energy } & \multicolumn{2}{|r|}{$19 \mathrm{~V}$} & \multicolumn{4}{|c|}{ Scan event 4. Selected Reaction Monitoring (SRM) } \\
\hline \multicolumn{2}{|c|}{ Repeat } & \multicolumn{2}{|c|}{ Top 2 precursors } & Scan time $0.5 \mathrm{~s}$ & \multicolumn{2}{|c|}{ Scan width 0.5} & CID $19 \mathrm{~V}$ \\
\hline \multicolumn{4}{|c|}{ Segment 2 (2-18 $\mathrm{min})$} & Precursor $(m / z)$ & Prc & lct $(m / z)$ & Analyte \\
\hline Scan event 1. F & ull-s & MS sam & as Segment 1 & 385.347 & & 7.337 & Vitamin $D_{3}$ \\
\hline Scan event 2. 1 & DDA & S/MS sam & e as Segment 1 & 397.347 & & 9.337 & Vitamin $D_{2}$ \\
\hline Scan event 3. $Q$ & $3 \mathrm{Se}$ & ted Ion $\mathrm{M}$ & onitoring (SIM) & 403.358 & & 7.122 & $\delta$-Tocopherol \\
\hline $0.5 \mathrm{~s}$ scan tim & & 0.5 & scan width & 416.365 & & 1.133 & $\gamma, \beta$-Tocopherol \\
\hline$m / z$ & & & Analyte & 417.373 & & 1.133 & $\gamma, \beta$-Tocopherol \\
\hline 269.227 & & & retinols & 431.389 & & 5.149 & $\alpha$-Tocopherol \\
\hline 287.238 & & Ret & (Vit. A) & 437.427 & & 167 & $d_{6}-\alpha-$ Tocopherol \\
\hline 301.217 & & $\operatorname{Re}$ & inoic Acid & $\mathrm{Se}$ & r & $(36-44 n$ & in) \\
\hline 329.248 & & Reti & nyl Acetate & Scan event 1. & $\underline{\mathrm{E}}$ & $\mathrm{n} \mathrm{MS} \mathrm{sam}$ & e as Segment 1 \\
\hline Scan event 4. S & ele & d Rxn. Mo & toring (SRM) & Scan event 2.1 & DD/ & 1S/MS san & ne as Segment 1 \\
\hline Scan time $0.5 \mathrm{~s}$ & Sca & Nidth 0.5 & CID $19 \mathrm{~V}$ & Scan event 3. C & $3 \mathrm{~s}$ & cted Ion N & lonitoring (SIM) \\
\hline Precursor $(m / z)$ & Prc & Ict $(m / z)$ & Analyte & $0.5 \mathrm{~s}$ scan tim & & & scan width \\
\hline 269.227 & & 3.070 & Retinol & $m / z$ & & & Analyte \\
\hline 301.217 & & 9.123 & Retinoic Acid & 451.358 & & Phylloc & yuinone (Vit. $\mathrm{K}_{1}$ ) \\
\hline 329.248 & & 9.227 & Ret. Acetate & 473.400 & & $\alpha$-Toc & pheryl Acetate \\
\hline Seg & $\mathrm{m}$ & $3(18-36 \mathrm{~m}$ & & Scan event 4. Se & lecte & Reaction I & Monitoring (SRM) \\
\hline Scan event 1. F & ull-s & n MS sam & as Segment 1 & Scan time $0.5 \mathrm{~s}$ & Sca & width 0.5 & CID $19 \mathrm{~V}$ \\
\hline Scan event 2. D & $\mathrm{DA}$ & /MS sam & as Segment 1 & Precursor $(m / z)$ & & uct $(m / z)$ & Analyte \\
\hline Scan event $3 . Q$ & $3 \mathrm{Se}$ & ted Ion $\mathrm{M}$ & onitoring (SIM) & 451.358 & & 7.240 & Vitamin $\mathrm{K}_{1}$ \\
\hline $0.5 \mathrm{~s}$ scan tim & & 0.5 & scan width & 473.400 & & 7.250 & $\alpha$-Toco. Acetate \\
\hline$m / z$ & & & Analyte & & $\mathrm{gm}$ & $5(44-54 n$ & nin) \\
\hline 385.347 & & Choleca & ciferol (Vit. $D_{3}$ ) & Identical to & Segn & tt 4 for th & ese analyses. \\
\hline 397.347 & & Ergocal & ciferol (Vit. $D_{2}$ ) & SIM and SF & $\mathrm{M} \mathrm{f}$ & $\beta$-Caroten & e removed. \\
\hline
\end{tabular}


Table S-10. TSQ Vantage EMR tandem sector quadrupole mass spectrometer (Thermo Fisher Scientific, San Jose, CA, USA). Multi-segment qualitative and SIM and SRM quantitative analysis parameters for ${ }^{1} D$ HPLC analysis.

\begin{tabular}{|c|c|c|}
\hline \multicolumn{3}{|c|}{ Cherry and Parinari Seed Oil Extract Analysis } \\
\hline \multicolumn{3}{|c|}{ APCI Source Parameters - Same as for FSV given in Table 9} \\
\hline \multicolumn{3}{|c|}{$\begin{array}{l}\text { 1.0 mTorr Argon CID gas turned on throughout all scans. All scans except precursor scans used Q3 with } \\
\text { 0.7 FWHM peak widths unless otherwise stated. All scans were in }(+) \text { ion centroid mode. }\end{array}$} \\
\hline Segment 1 (0-2 min) & \multicolumn{2}{|c|}{ Segment 5 (44-130 min) } \\
\hline Scan event 1. Same Full-Scan MS as for FSV. & \multicolumn{2}{|c|}{ Scan event 1. Same Full-Scan MS as for FSV. } \\
\hline Scan event 2. Same 1 DDA MS/MS as for FSV. & \multicolumn{2}{|c|}{ Scan event 2. Narrow range full-scan MS of TAGs } \\
\hline Scan event 3. Same DDA MS/MS as for FSV. & Scan range & $m / z 800-1100$ \\
\hline Segment 2 (2-18 $\mathrm{min})$ & Scan time & $0.40 \mathrm{~s}$ \\
\hline Scan event 1. Same Full-Scan MS as for FSV. & \multicolumn{2}{|c|}{ Scan event 3. DDA MS/MS of TAGs } \\
\hline Scan event 2. Same 1 DDA MS/MS as for FSV. & Signal threshold & $1 \mathrm{e} 4$ \\
\hline Scan event 3. Same 4 x SIM as for FSV. & Scan time & $1.0 \mathrm{~s}$ \\
\hline Scan event 4. Same $3 \times$ SRM as for FSV. & Collision energy & $30 \mathrm{~V}$ \\
\hline Segment 3 (18-36 min) & Repeat & Top precursor only \\
\hline Scan event 1. Same Full-Scan MS as for FSV. & \multicolumn{2}{|c|}{ Scan event 4. Narrow range MS of [DAG] ${ }^{+}$} \\
\hline Scan event 2. Same 1 DDA MS/MS as for FSV. & Scan range & $m / z 400-750$ \\
\hline Scan event 3. Same 7 x SIM as for FSV. & Scan time & $0.45 \mathrm{~s}$ \\
\hline Scan event 4. Same $7 \times$ SRM as for FSV. & \multicolumn{2}{|c|}{ Scan event 5. DDA MS/MS of [DAG] ${ }^{+}$} \\
\hline Segment 4 (36-44 min) & Signal threshold & $1 \mathrm{e} 4$ \\
\hline Scan event 1. Same Full-Scan MS as for FSV. & Scan time & $0.75 \mathrm{~s}$ \\
\hline Scan event 2. Same 1 DDA MS/MS as for FSV. & Collision energy & $40 \mathrm{~V}$ \\
\hline Scan event 3. Same $2 \times$ SIM as for FSV. & Repeat & Top precursor only \\
\hline Scan event 4. Same $2 \times$ SRM as for FSV. & \multicolumn{2}{|c|}{ Segment 5. 54-130 min for Soybean Oil only. } \\
\hline
\end{tabular}

\section{B) ${ }^{2}$ D UHPLC detection.}

Table S-11. TSQ Quantum Access Max tandem sector quadrupole mass spectrometer (Thermo Fisher Scientific, San Jose, CA, USA). Full-scan MS for ${ }^{2}$ D UHPLC qualitative analysis.

\begin{tabular}{|c|c|}
\hline \multicolumn{2}{|c|}{ Fat-Soluble Vitamin and Seed Oil Extract Analysis } \\
\hline \multicolumn{2}{|c|}{ APPI Source Parameters } \\
\hline Parameter & Setting \\
\hline Vaporizer heater & $400{ }^{\circ} \mathrm{C}$ \\
\hline Sheath gas (Nitrogen) & 60 \\
\hline Auxiliary gas (Nitrogen) & 5 \\
\hline Sweep gas & 0 \\
\hline Capillary temp. & $270{ }^{\circ} \mathrm{C}$ \\
\hline Skimmer offset & $10 \mathrm{~V}$ \\
\hline
\end{tabular}

1.0 mTorr Argon CID gas turned on throughout all scans. All scans except precursor scans used Q3. All used 0.7 FWHM peak widths unless otherwise stated. All scans were in (+) ion centroid mode.

All runs use acetone dopant at $20 \mu \mathrm{L} / \mathrm{min}$ by Applied Biosystems AB140B dual piston syringe pump.

\begin{tabular}{|c|c|c|c|}
\hline \multicolumn{2}{|c|}{ Scan event 1. Q3 Full-scan MS } & \multicolumn{2}{|c|}{ Scan events 2. Data-dependent MS/MS } \\
\hline Scan range & $\mathrm{m} / \mathrm{z}$ 150-2000 & Signal threshold & $1 \mathrm{e} 4$ \\
\hline Scan time & $1.0 \mathrm{~s}$ & Scan time & $0.9 \mathrm{~s}$ \\
\hline \multicolumn{2}{|c|}{ Runt time 53.50 min for FSVs. } & Collision energy & $50 \mathrm{~V}$ \\
\cline { 2 - 4 } Run time 129.50 min for seed oil extracts. & Repeat & Top precursor only \\
\hline
\end{tabular}


Table S-12. LCQ Deca XP hyperbolic ion trap mass spectrometer (Thermo Fisher Scientific, San Jose, CA, USA). Full-scan MS for ${ }^{2} D$ UHPLC qualitative analysis.

\begin{tabular}{|c|c|c|c|}
\hline \multicolumn{4}{|c|}{ Fat-Soluble Vitamin and Seed Oil Extract Analysis } \\
\hline \multicolumn{4}{|c|}{ APPI Source Parameters } \\
\hline \multicolumn{2}{|c|}{ Parameter } & \multicolumn{2}{|c|}{ Setting } \\
\hline \multicolumn{2}{|c|}{ Sheath gas (Nitrogen) } & \multicolumn{2}{|c|}{35} \\
\hline \multicolumn{2}{|c|}{ Auxiliary gas (Nitrogen) } & \multicolumn{2}{|c|}{5} \\
\hline \multicolumn{2}{|c|}{ Capillary temp. } & \multicolumn{2}{|c|}{$265^{\circ} \mathrm{C}$} \\
\hline \multicolumn{2}{|c|}{ Spray Voltage } & \multicolumn{2}{|c|}{$5000 \mathrm{~V}$} \\
\hline \multicolumn{4}{|c|}{$\begin{array}{l}\text { All scans were in (+) ion mode with centroided masses. } \\
\text { All runs use ammonium formate }\left(\mathrm{NH}_{4} \mathrm{OCOH}\right) \text { in methanol }(1: 4) \text { at } 20 \mu \mathrm{L} / \mathrm{min} \\
\text { supplied by Applied Biosystems } \mathrm{AB140C} \text { dual piston syringe pump. }\end{array}$} \\
\hline \multicolumn{2}{|c|}{ Scan event 1. Full-scan MS } & \multicolumn{2}{|c|}{ Scan events 2\&3. Data-dependent MS/MS } \\
\hline Scan range & $m / z 200-1050$ & Signal threshold & $1 \mathrm{e} 4$ \\
\hline Scan time & $1.0 \mathrm{~s}$ & Precursor range & $\mathrm{m} / \mathrm{z} 200-1050$ \\
\hline \multirow{5}{*}{\multicolumn{2}{|c|}{$\begin{array}{l}\text { Runt time } 54.0 \text { min for FSVs. } \\
\text { Run time } 130.0 \text { min for seed oil extracts. }\end{array}$}} & Norm. Coll. Energy & $45.0 \%$ \\
\hline & & Activation $\mathrm{Q}$ & 0.350 \\
\hline & & Activation time & $900 \mathrm{~ms}$ \\
\hline & & Isolation width & $2.0 \mathrm{~m} / \mathrm{z}$ \\
\hline & & Repeat & Top 2 precursor \\
\hline
\end{tabular}

5. External standard results for tocopherols by APCI-MS, internal standard and external standard results by UV detection.

Table S-13. Internal and external standard results by UV detection and external standard results by SIM and SRM APCI-MS from ${ }^{1}$ D HPLC, in ppm (= $\mu \mathrm{g} / \mathrm{g}$ oil).

\begin{tabular}{|c|c|c|c|c|c|c|c|c|c|c|}
\hline & \multicolumn{10}{|c|}{ Internal Standard Method } \\
\hline & & $\alpha$-Toco & SD & $r^{2}$ & $\gamma$-Toco & SD & $r^{2}$ & $\delta$-Toco & SD & $r^{2}$ \\
\hline Cherry & UV & 217 & 22 & 0.9765 & 636 & 14 & 0.9662 & 151 & 10 & 0.9893 \\
\hline Parinari & UV & $5427^{a}$ & 104 & & 616 & 31 & & & & \\
\hline \multirow[t]{3}{*}{ Soybean } & UV & & & & 193 & 19 & & 205 & 12 & \\
\hline & \multicolumn{10}{|c|}{ External Standard Method } \\
\hline & & $\alpha$-Toco & SD & $r^{2}$ & $\gamma$-Toco & SD & $r^{2}$ & $\delta$-Toco & SD & $r^{2}$ \\
\hline \multirow[t]{3}{*}{ Cherry } & UV & 217 & 22 & 0.9765 & 717 & 17 & 0.9738 & 118 & 13 & 0.9947 \\
\hline & SIM & 278 & 18 & 0.9898 & 583 & 13 & 0.9766 & 98.0 & 5.1 & 0.9762 \\
\hline & SRM & 244 & 21 & 0.9328 & 717 & 40 & 0.9459 & 126 & 10 & 0.9324 \\
\hline \multirow[t]{3}{*}{ Parinari } & UV & $5427^{a}$ & 104 & & 688 & 39 & & & & \\
\hline & SIM & 643.3 & 1.6 & & 705 & 27 & & & & \\
\hline & SRM & 631 & 95 & & 849 & 127 & & & & \\
\hline \multirow[t]{3}{*}{ Soybean } & UV & & & & 168 & 24 & & 186 & 15 & \\
\hline & SIM & 30.8 & 1.8 & & 150.8 & 4.3 & & 213 & 11 & \\
\hline & SRM & 42.0 & 1.2 & & 174.2 & 6.9 & & 187 & 17 & \\
\hline
\end{tabular}


Table S-14. Limits of detection (LOD) and limits of quantification (LOQ) calculated from calibration lines. Observed LOD and LOQ (e.g. from sequential dilution) are likely lower.

\begin{tabular}{|c|c|c|c|c|}
\hline & \multicolumn{4}{|c|}{ Internal Standard Method } \\
\hline & & $\alpha$-Toco (ppm) & $\gamma$-Toco (ppm) & $\delta$-Toco (ppm) \\
\hline LOD $^{\mathbf{a}}$ & UV & 200 & 15 & 44 \\
\hline$b+3^{*} \sigma_{0.125}$ & SIM & 7.5 & 79 & 99 \\
\hline & SRM & 6.5 & 42 & 23 \\
\hline LOQ $^{\mathbf{b}}$ & UV & 668 & 51 & 146 \\
\hline$b+10^{*} \sigma_{0.125}$ & SIM & 25 & 264 & 330 \\
\hline & SRM & 22 & 139 & 76 \\
\hline & & External Standard Method & $\delta$-Toco (ppm) \\
\hline LOD $^{\mathbf{a}}$ & UV & $\alpha$-Toco (ppm) & $\gamma$-Toco (ppm) & 71 \\
\hline$b+3^{*} \sigma_{0.125}$ & SIM & 200 & 36 & 13 \\
\hline & SRM & 25 & 59 & 235 \\
\hline LOQ $^{\mathbf{b}}$ & UV & \multicolumn{4}{|c|}{668} & 39 & 271 \\
\hline$b+10^{*} \sigma_{0.125}$ & SIM & 82 & 120 & 42 \\
\hline & SRM & 68 & 131 & \\
\hline
\end{tabular}

a Limit of Detection (LOD) area (E.S.) or ratio (I.S.) = (intercept $(b)+3 \times$ standard deviation of lowest standard $[0.125 \mu \mathrm{g} / \mathrm{mL}]$ ) put into calibration equation and converted to $\mathrm{ppm}(=\mu \mathrm{g} / \mathrm{g}$ oil).

bimit of Quantification (LOQ) area (E.S.) or ratio (I.S.) $=$ (intercept $(b)+10 \mathrm{x}$ standard deviation of lowest standard $[0.125 \mu \mathrm{g} / \mathrm{mL}])$ put into calibration equation and converted to $\mathrm{ppm}(=\mu \mathrm{g} / \mathrm{g}$ oil).

The results for UV detection of $\alpha$-tocopherol in Table S-14 demonstrate the shortcomings of UV detection in real samples. Any other sterols or other molecules that absorb at all at the wavelength of $297 \mathrm{~nm}$ in the time range around $33.8 \mathrm{~min}$ will contribute to the peak area attributed to $\alpha$-tocopherol. For instance, the second peak of di-eleosteroyl diacylglycerol, EIEI $=m / z 595.5$ [DAG] ${ }^{+}$and $m / z 613.5$ $[\mathrm{M}+\mathrm{H}]^{+}$, eluted overlapped with $\alpha$-tocopherol (both at $33.78 \mathrm{~min}$ by APCI-MS) and was present in a much larger amount than $\alpha$-tocopherol. Therefore, even a small absorbance of 18:3 at $297 \mathrm{~nm}$ would skew the results for $\alpha$-tocopherol, as observed. By both SIM and SRM APCI-MS this overlap presented no problem.

It is important to note that both internal standard and external standard UV and APCI-MS approaches showed higher levels of tocopherols than were observed in the results from the commercial labs previously presented. Thus, all of these approaches, when coupled to an extract-and-shoot sample preparation approach, identified larger amounts of tocopherols than were found using the commonly used heated saponification, extraction and collection, followed by derivatization approach widely used. ${ }^{3}$ Even when cherry kernels were analyzed without saponification and derivatization, the amount of tocopherols varied greatly with extraction conditions, ${ }^{4}$ with the extraction of Folch et al. ${ }^{1}$ commonly considered the 'gold standard'. ${ }^{5}$ 
Of course, as we previously reported with vitamin D, UV data should not be trusted without MS data to prove that the peaks are pure. And if not pure, as in the case of $\alpha$-tocopherol, UV data should not be used for quantification.

On the other hand, as with vitamin $D,{ }^{6}$ if $\mathrm{MS}$ data can be used to prove that the peaks are pure, UV data may be more sensitive and show lower standard deviations than APCI-MS, since APCI inherently has more chemical noise associated with the ionization process. This is demonstrated by the lower LOD and LOQ for $\gamma$-tocopherol in Table S-14, since these values are based on the standard deviation in the lowest standard $(0.125 \mu \mathrm{g} / \mathrm{mL})$.

\section{Detailed compositions of FAMEs for PSO, CPO, and SBO.}

Table S-15. Detailed response factor normalized and GC-FID fatty acid compositions of Parinari curatellifolia seed oil, including compositions of isomers of 18:3 (CN:db) and oxo-eleostearic acid.

\begin{tabular}{|c|c|c|c|c|c|c|}
\hline & \multicolumn{3}{|c|}{ Parinari Seed Oil (PSO) } & & & \\
\hline & APPI-MS & SD & GC-FID & & & \\
\hline$M(14: 0)$ & $0.000 \%$ & $0.000 \%$ & $0.033 \%$ & & & \\
\hline Po (16:1) & $0.020 \%$ & $0.001 \%$ & $0.018 \%$ & & & \\
\hline$P(16: 0)$ & $9.136 \%$ & $0.042 \%$ & $9.287 \%$ & & GC-FID & SD \\
\hline $\begin{array}{c}\text { EI } \\
\text { (all 18:3) }\end{array}$ & $48.768 \%$ & $0.096 \%$ & $48.840 \%$ & $\alpha-E I$ & $90.00 \%$ & $0.07 \%$ \\
\hline L (18:2) & $14.379 \%$ & $0.137 \%$ & $13.992 \%$ & $\beta$-El & $7.63 \%$ & $0.06 \%$ \\
\hline $\mathrm{O}(18: 1)$ & $17.709 \%$ & $0.060 \%$ & $17.518 \%$ & Ln & $0.09 \%$ & $0.00 \%$ \\
\hline$S(18: 0)$ & $7.267 \%$ & $0.113 \%$ & $7.589 \%$ & $18: 3 \times 1$ & $1.70 \%$ & $0.04 \%$ \\
\hline$A(20: 0)$ & $0.402 \%$ & $0.006 \%$ & $0.395 \%$ & $18: 3 \times 2$ & $0.38 \%$ & $0.01 \%$ \\
\hline G $(20: 1)$ & $0.688 \%$ & $0.014 \%$ & $0.695 \%$ & $18: 3 \times 3$ & $0.14 \%$ & $0.01 \%$ \\
\hline 21:0 & $0.003 \%$ & $0.000 \%$ & $0.000 \%$ & $18: 3 \times 4$ & $0.06 \%$ & $0.01 \%$ \\
\hline B $(22: 0)$ & $0.031 \%$ & $0.000 \%$ & $0.032 \%$ & & $100.00 \%$ & \\
\hline 23:0 & $0.005 \%$ & $0.000 \%$ & $0.014 \%$ & & GC-FID & SD \\
\hline $\operatorname{Lg}(24: 0)$ & $0.022 \%$ & $0.001 \%$ & $0.033 \%$ & oxo-El-1 & $3.60 \%$ & $0.49 \%$ \\
\hline 25:0 & $0.004 \%$ & $0.000 \%$ & $0.012 \%$ & Oxo-El-2 & $81.01 \%$ & $0.76 \%$ \\
\hline $\mathrm{Ce}(26: 0)$ & $0.003 \%$ & $0.000 \%$ & $0.000 \%$ & oxo-El-3 & $2.07 \%$ & $0.34 \%$ \\
\hline OXO-El & $1.563 \%$ & $0.017 \%$ & $1.543 \%$ & OXo-El-4 & $13.32 \%$ & $0.30 \%$ \\
\hline Sum & $100.000 \%$ & & $100.000 \%$ & & $100.00 \%$ & \\
\hline
\end{tabular}


Table S-16. Detailed response factor normalized and GC-FID fatty acid compositions of cherry pit oil (not including isomers).

\begin{tabular}{c|ccc|}
\hline \multicolumn{4}{|c}{ Cherry Pit Oil (CPO) } \\
\hline FA & APCI-MS & SD & GC-FID \\
\hline M & $0.006 \%$ & $0.000 \%$ & $0.069 \%$ \\
Po & $0.629 \%$ & $0.030 \%$ & $0.626 \%$ \\
P & $8.841 \%$ & $0.120 \%$ & $8.324 \%$ \\
EI & $5.766 \%$ & $0.115 \%$ & $5.760 \%$ \\
L & $32.650 \%$ & $0.243 \%$ & $33.320 \%$ \\
O & $47.825 \%$ & $0.203 \%$ & $47.598 \%$ \\
S & $2.437 \%$ & $0.011 \%$ & $2.507 \%$ \\
A & $0.943 \%$ & $0.008 \%$ & $0.916 \%$ \\
G & $0.423 \%$ & $0.002 \%$ & $0.405 \%$ \\
21 & $0.015 \%$ & $0.001 \%$ & $0.015 \%$ \\
B & $0.208 \%$ & $0.001 \%$ & $0.202 \%$ \\
23 & $0.025 \%$ & $0.001 \%$ & $0.031 \%$ \\
Lg & $0.193 \%$ & $0.006 \%$ & $0.187 \%$ \\
25 & $0.017 \%$ & $0.001 \%$ & $0.022 \%$ \\
Ce & $0.022 \%$ & $0.001 \%$ & $0.018 \%$ \\
\hline Sum & $100.000 \%$ & & $100.000 \%$
\end{tabular}

Table S-17. Detailed response factor normalized and GC-FID fatty acid compositions of soybean oil (not including isomers, discussed below).

\begin{tabular}{c|ccc|}
\hline \multicolumn{4}{|c|}{ Soybean Oil (SBO) } \\
\hline FA & APCI-MS & SD & GC-FID \\
\hline M & $0.002 \%$ & $0.000 \%$ & $0.094 \%$ \\
Po & $0.098 \%$ & $0.009 \%$ & $0.102 \%$ \\
P & $12.109 \%$ & $0.064 \%$ & $11.953 \%$ \\
Ln & $7.406 \%$ & $0.103 \%$ & $7.519 \%$ \\
L & $52.699 \%$ & $0.474 \%$ & $51.515 \%$ \\
0 & $22.369 \%$ & $0.189 \%$ & $23.619 \%$ \\
S & $4.286 \%$ & $0.113 \%$ & $4.175 \%$ \\
A & $0.336 \%$ & $0.008 \%$ & $0.323 \%$ \\
G & $0.197 \%$ & $0.005 \%$ & $0.184 \%$ \\
21 & $0.023 \%$ & $0.000 \%$ & $0.021 \%$ \\
B & $0.341 \%$ & $0.012 \%$ & $0.329 \%$ \\
23 & $0.016 \%$ & $0.000 \%$ & $0.032 \%$ \\
Lg & $0.106 \%$ & $0.005 \%$ & $0.102 \%$ \\
25 & $0.005 \%$ & $0.000 \%$ & $0.008 \%$ \\
Ce & $0.007 \%$ & $0.001 \%$ & $0.021 \%$ \\
\hline Sum & $100.000 \%$ & & $100.000 \%$
\end{tabular}

GC-FID FA compositions were determined from the weight percentage of fatty acid methyl esters (FAME) and converted from weight percent FAME to mole percent FA.

As expected, the composition in Table S-16 is in excellent agreement to the FA composition given for CPO previously. ${ }^{2}$ Similarly, the FA composition for wild SBO (Glycine soja) is in excellent agreement with the composition of SBO from a dietary supplement recently reported using the 'dilute-and-shoot' approach with quadruple parallel mass spectrometry. ${ }^{7}$ 


\section{Detailed compositions of diacylglycerols for CPO, PSO, and SBO.}

Table S-18. Diacylglyerol (DAG) composition of $\mathrm{CPO}$ by response factor normalized APCI-MS.

\begin{tabular}{|c|c|c|c|}
\hline \multicolumn{4}{|c|}{ Cherry Pit Oil (CPO) } \\
\hline DAG & \% Comp. & SD & $2 / 1$ \\
\hline EIEI & $0.03 \%$ & $0.01 \%$ & 0.62 \\
\hline LEI & $1.11 \%$ & $0.07 \%$ & 0.66 \\
\hline LL & $18.14 \%$ & $0.56 \%$ & 0.47 \\
\hline OEI & $0.54 \%$ & $0.02 \%$ & 0.54 \\
\hline PoO & $0.19 \%$ & $0.04 \%$ & 0.64 \\
\hline PoP & $0.57 \%$ & $0.10 \%$ & 0.54 \\
\hline OL & $28.72 \%$ & $1.62 \%$ & 0.35 \\
\hline PL & $12.06 \%$ & $1.06 \%$ & 0.23 \\
\hline 00 & $22.40 \%$ & $1.14 \%$ & 0.28 \\
\hline OP & $11.38 \%$ & $0.13 \%$ & 0.28 \\
\hline SL & $2.98 \%$ & $0.09 \%$ & 0.29 \\
\hline OS & $1.89 \%$ & $1.64 \%$ & 0.26 \\
\hline Sum & $100.00 \%$ & & \\
\hline DAG/TAG & $1.08 \%$ & $\pm 0.03 \%$ & \\
\hline
\end{tabular}

Table S-20. Diacylglycerol (DAG) composition of SBO by response factor normalized $\mathrm{APCl}$ MS.

\begin{tabular}{c|ccc|}
\hline \multicolumn{4}{|c|}{ Soybean Oil (SBO) } \\
\hline DAG & \% Comp. & SD & $\mathbf{2 / 1}$ \\
\hline LnLn & $0.49 \%$ & $0.07 \%$ & 0.34 \\
LLn & $7.29 \%$ & $0.19 \%$ & 0.54 \\
LL & $43.73 \%$ & $1.39 \%$ & 0.56 \\
OLn & $1.02 \%$ & $0.18 \%$ & 0.40 \\
PoO & $0.00 \%$ & $0.00 \%$ & \\
PoP & $0.03 \%$ & $0.01 \%$ & 0.71 \\
OL & $15.84 \%$ & $1.20 \%$ & 0.39 \\
PL & $15.78 \%$ & $0.37 \%$ & 0.20 \\
OO & $4.90 \%$ & $0.22 \%$ & 0.26 \\
OP & $3.93 \%$ & $0.07 \%$ & 0.29 \\
SL & $5.33 \%$ & $0.10 \%$ & 0.27 \\
OS & $1.67 \%$ & $0.08 \%$ & 0.26 \\
Sum & $100.00 \%$ & & \\
DAG/TAG & $1.39 \%$ & $\pm 0.04 \%$ &
\end{tabular}

Table S-19. More detailed diacylglycerol (DAG) composition of Parinari curatellifolia seed oil extract by response factor normalized APCI-MS.

\begin{tabular}{c|ccc|}
\hline \multicolumn{4}{|c|}{ Parinari Seed Oil (PSO) } \\
\hline DAG & \% Comp. & SD & $\mathbf{2} / \mathbf{1}$ \\
\hline oxEloxEI & $0.02 \%$ & $0.00 \%$ & \\
oxEoEo & $0.75 \%$ & $0.07 \%$ & 0.21 \\
oxEoL & $0.10 \%$ & $0.01 \%$ & 0.28 \\
oxEoO & $0.17 \%$ & $0.01 \%$ & $\mathbf{0 . 2 3}$ \\
oxEoP & $0.10 \%$ & $0.01 \%$ & 0.30 \\
EIEI & $17.91 \%$ & $0.62 \%$ & 2.57 \\
LEI & $11.25 \%$ & $0.10 \%$ & 2.72 \\
LL & $11.49 \%$ & $0.10 \%$ & 4.19 \\
OEI & $12.60 \%$ & $0.30 \%$ & 1.57 \\
PoO & $0.75 \%$ & $0.03 \%$ & 1.61 \\
PoP & $0.06 \%$ & $0.01 \%$ & 1.37 \\
OL & $14.53 \%$ & $0.45 \%$ & 3.43 \\
PL & $6.73 \%$ & $0.10 \%$ & 1.53 \\
OO & $6.87 \%$ & $0.17 \%$ & 1.50 \\
OP & $6.91 \%$ & $0.11 \%$ & 0.72 \\
SL & $4.72 \%$ & $0.17 \%$ & 1.59 \\
OS & $5.03 \%$ & $0.14 \%$ & 0.52 \\
\hline Sum & $100.00 \%$ & & \\
DAG/TAG & $1.52 \%$ & $\pm 0.05 \%$ & \\
\hline
\end{tabular}


8. Detailed compositions of TAGs for CPO, PSO, and SBO.

Table S-21. Detailed triacylglycerol (TAG) composition of CPO by response factor normalized APCI-MS. Structures shown here are not regiospecific.

\begin{tabular}{|c|c|c|c|c|c|c|c|}
\hline \multicolumn{8}{|c|}{ Cherry Pit Oil (CPO) } \\
\hline TAG & RT & \% Comp. & SD & TAG & RT & \% Comp. & SD \\
\hline EIEIEI & 55.10 & $0.017 \%$ & $0.001 \%$ & LOA & 94.47 & $0.880 \%$ & $0.021 \%$ \\
\hline EIEIL & 56.80 & $0.634 \%$ & $0.012 \%$ & LLB & 94.98 & $0.153 \%$ & $0.002 \%$ \\
\hline LLEl & 58.46 & $5.377 \%$ & $0.066 \%$ & POS & 95.97 & $0.380 \%$ & $0.003 \%$ \\
\hline LLL & 60.33 & $3.964 \%$ & $0.025 \%$ & PLA & 96.46 & $0.218 \%$ & $0.009 \%$ \\
\hline EIEIO & 62.14 & $0.211 \%$ & $0.008 \%$ & SSL & 96.54 & $0.112 \%$ & $0.003 \%$ \\
\hline EIEIP & 63.32 & $0.040 \%$ & $0.003 \%$ & OL-21 & 98.76 & $0.019 \%$ & $0.001 \%$ \\
\hline OLEI & 64.22 & $4.258 \%$ & $0.275 \%$ & LL-23 & 99.18 & $0.024 \%$ & $0.000 \%$ \\
\hline PLEI & 65.50 & $2.044 \%$ & $0.084 \%$ & PPS & 99.76 & $0.001 \%$ & $0.000 \%$ \\
\hline LLO & 66.35 & $13.434 \%$ & $0.372 \%$ & LEILg & 100.66 & $0.010 \%$ & $0.000 \%$ \\
\hline PoPL & 67.27 & $0.902 \%$ & $0.110 \%$ & SOG & 101.77 & $0.018 \%$ & $0.005 \%$ \\
\hline LLP & 67.76 & $3.506 \%$ & $0.044 \%$ & OOA & 102.36 & $0.755 \%$ & $0.015 \%$ \\
\hline EIEIG & 68.73 & $0.002 \%$ & $0.000 \%$ & OLB & 102.64 & $0.178 \%$ & $0.003 \%$ \\
\hline MOL & 69.53 & $0.019 \%$ & $0.001 \%$ & LLLg & 102.95 & $0.140 \%$ & $0.003 \%$ \\
\hline EoEos & 71.12 & $0.028 \%$ & $0.002 \%$ & POA & 104.27 & $0.202 \%$ & $0.001 \%$ \\
\hline OOEI & 71.12 & $2.090 \%$ & $0.017 \%$ & SSO & 104.37 & $0.130 \%$ & $0.002 \%$ \\
\hline POEI & 72.73 & $0.614 \%$ & $0.009 \%$ & PBL & 104.42 & $0.058 \%$ & $0.001 \%$ \\
\hline OOL & 73.73 & $16.632 \%$ & $0.332 \%$ & SLA & 104.68 & $0.060 \%$ & $0.002 \%$ \\
\hline LLG & 73.86 & $0.318 \%$ & $0.016 \%$ & $00-21$ & 105.87 & $0.012 \%$ & $0.002 \%$ \\
\hline POPo & 74.81 & $0.992 \%$ & $0.117 \%$ & OL-23 & 106.04 & $0.028 \%$ & $0.001 \%$ \\
\hline POL & 75.42 & $7.442 \%$ & $0.144 \%$ & LL-25 & 106.25 & $0.016 \%$ & $0.001 \%$ \\
\hline LLS & 76.40 & $0.774 \%$ & $0.018 \%$ & OOB & 108.92 & $0.148 \%$ & $0.005 \%$ \\
\hline PPL & 77.30 & $0.597 \%$ & $0.012 \%$ & OLLg & 109.10 & $0.167 \%$ & $0.003 \%$ \\
\hline LEIA & 79.60 & $0.027 \%$ & $0.001 \%$ & LLCe & 109.28 & $0.017 \%$ & $0.000 \%$ \\
\hline EIEIA & 80.13 & $0.002 \%$ & $0.000 \%$ & PLLg & 110.63 & $0.052 \%$ & $0.004 \%$ \\
\hline SOEI & 82.46 & $0.989 \%$ & $0.033 \%$ & РОВ & 110.65 & $0.046 \%$ & $0.002 \%$ \\
\hline 000 & 82.51 & $17.235 \%$ & $0.247 \%$ & SLB & 110.83 & $0.020 \%$ & $0.001 \%$ \\
\hline OLG & 82.59 & $0.477 \%$ & $0.004 \%$ & SOA & 110.89 & $0.057 \%$ & $0.004 \%$ \\
\hline OOP & 84.44 & $6.991 \%$ & $0.153 \%$ & $00-23$ & 111.81 & $0.023 \%$ & $0.001 \%$ \\
\hline PLG & 84.54 & $0.193 \%$ & $0.015 \%$ & OL-25 & 111.95 & $0.018 \%$ & $0.000 \%$ \\
\hline SLO & 85.28 & $1.695 \%$ & $0.011 \%$ & OOLg & 114.76 & $0.152 \%$ & $0.009 \%$ \\
\hline LLA & 86.08 & $0.622 \%$ & $0.009 \%$ & OLCe & 114.83 & $0.020 \%$ & $0.002 \%$ \\
\hline POP & 86.51 & $0.606 \%$ & $0.001 \%$ & PLCe & 116.40 & $0.005 \%$ & $0.000 \%$ \\
\hline SLP & 87.36 & $0.309 \%$ & $0.003 \%$ & POLg & 116.42 & $0.042 \%$ & $0.002 \%$ \\
\hline PPP & 89.98 & $0.001 \%$ & $0.000 \%$ & SLLg & 116.79 & $0.009 \%$ & $0.001 \%$ \\
\hline LL-21 & 90.60 & $0.016 \%$ & $0.001 \%$ & SOB & 116.82 & $0.011 \%$ & $0.002 \%$ \\
\hline OOG & 91.14 & $0.270 \%$ & $0.002 \%$ & $00-25$ & 117.64 & $0.016 \%$ & $0.002 \%$ \\
\hline EIOA & 91.70 & $0.027 \%$ & $0.003 \%$ & OOCe & 120.56 & $0.018 \%$ & $0.001 \%$ \\
\hline LEIB & 92.27 & $0.012 \%$ & $0.000 \%$ & POCe & 122.44 & $0.006 \%$ & $0.002 \%$ \\
\hline OOS & 93.82 & $2.332 \%$ & $0.005 \%$ & SOLg & 122.79 & $0.011 \%$ & $0.001 \%$ \\
\hline EoSS & 93.98 & $0.066 \%$ & $0.020 \%$ & OOMо & 126.67 & $0.001 \%$ & $0.000 \%$ \\
\hline & & & & Sum & & $99.999 \%$ & \\
\hline
\end{tabular}


Table S-22. Detailed triacylglycerol (TAG) composition of PSO by response factor normalized APCI-MS. TAG structures given are not regiospecific.

\begin{tabular}{|c|c|c|c|c|c|c|c|}
\hline \multicolumn{8}{|c|}{ Parinari Seed Oil (PSO) } \\
\hline TAG & RT & \% Comp. & SD & TAG & RT & \% Comp. & SD \\
\hline $\begin{array}{c}\text { oxEloxEloxEl } \\
\text { oxEloxEIEI }\end{array}$ & $\begin{array}{l}32.02 \\
40.28\end{array}$ & $\begin{array}{l}0.000 \% \\
0.030 \%\end{array}$ & $\begin{array}{l}0.000 \% \\
0.001 \%\end{array}$ & $\begin{array}{c}\text { EIEl-21 } \\
\text { SLO }\end{array}$ & $\begin{array}{l}84.80 \\
85.04\end{array}$ & $\begin{array}{l}0.008 \% \\
1.392 \%\end{array}$ & $\begin{array}{l}0.000 \% \\
0.062 \%\end{array}$ \\
\hline oxEloxEIL & 41.35 & $0.005 \%$ & $0.000 \%$ & LLA & 85.83 & $0.026 \%$ & $0.001 \%$ \\
\hline oxEloxEIO & 43.58 & $0.011 \%$ & $0.000 \%$ & POP & 86.29 & $0.528 \%$ & $0.018 \%$ \\
\hline oxEloxEIP & 43.91 & $0.011 \%$ & $0.001 \%$ & SLP & 87.09 & $0.930 \%$ & $0.026 \%$ \\
\hline OXEIEIEI & 46.02 & $1.269 \%$ & $0.019 \%$ & ElEIB & 89.30 & $0.032 \%$ & $0.001 \%$ \\
\hline OXEIEIL & 47.08 & $0.384 \%$ & $0.009 \%$ & PPP & 89.64 & $0.022 \%$ & $0.000 \%$ \\
\hline oxEloxEIS & 47.38 & $0.013 \%$ & $0.000 \%$ & OOG & 90.98 & $0.071 \%$ & $0.001 \%$ \\
\hline oxEILL & 48.20 & $0.034 \%$ & $0.002 \%$ & EIOA & 91.46 & $0.122 \%$ & $0.010 \%$ \\
\hline OXEIEIO & 50.54 & $0.865 \%$ & $0.006 \%$ & LEIB & 92.04 & $0.010 \%$ & $0.000 \%$ \\
\hline OXEIEIP & 51.23 & $0.676 \%$ & $0.022 \%$ & EIEI-23 & 93.65 & $0.011 \%$ & $0.000 \%$ \\
\hline OXEILO & 51.87 & $0.085 \%$ & $0.001 \%$ & oos & 93.68 & $0.845 \%$ & $0.026 \%$ \\
\hline OXEILP & 52.56 & $0.043 \%$ & $0.001 \%$ & EISS & 93.83 & $0.934 \%$ & $0.023 \%$ \\
\hline OXEIOO & 56.21 & $0.143 \%$ & $0.004 \%$ & LLB & 94.84 & $0.003 \%$ & $0.000 \%$ \\
\hline OXEIEIS & 56.26 & $0.850 \%$ & $0.018 \%$ & POS & 95.79 & $0.747 \%$ & $0.009 \%$ \\
\hline OXEIOP & 56.83 & $0.058 \%$ & $0.000 \%$ & PLA & 96.36 & $0.136 \%$ & $0.001 \%$ \\
\hline OxEIPP & 57.75 & $0.008 \%$ & $0.000 \%$ & SSL & 96.38 & $0.400 \%$ & $0.012 \%$ \\
\hline oxEILS & 57.85 & $0.047 \%$ & $0.003 \%$ & ElEILg & 97.90 & $0.028 \%$ & $0.001 \%$ \\
\hline oxEIOS & 63.36 & $0.060 \%$ & $0.004 \%$ & OL-21 & 98.41 & $0.001 \%$ & $0.000 \%$ \\
\hline oxEISP & 64.23 & $0.038 \%$ & $0.001 \%$ & LL-23 & 98.96 & $0.001 \%$ & $0.000 \%$ \\
\hline OxEISS & 72.40 & $0.019 \%$ & $0.002 \%$ & PPS & 99.31 & $0.051 \%$ & $0.001 \%$ \\
\hline EIEIEl & 55.11 & $12.036 \%$ & $0.191 \%$ & LEILg & 100.40 & $0.009 \%$ & $0.000 \%$ \\
\hline EIEIL & 56.70 & $8.252 \%$ & $0.143 \%$ & SOG & 101.83 & $0.043 \%$ & $0.007 \%$ \\
\hline LLEI & 58.47 & $3.348 \%$ & $0.092 \%$ & ElEl-25 & 101.83 & $0.008 \%$ & $0.000 \%$ \\
\hline LLL & 60.31 & $0.405 \%$ & $0.013 \%$ & OOA & 102.10 & $0.030 \%$ & $0.002 \%$ \\
\hline EIEIO & 62.05 & $11.267 \%$ & $0.153 \%$ & OLB & 102.46 & $0.015 \%$ & $0.001 \%$ \\
\hline EIEIP & 63.23 & $8.090 \%$ & $0.141 \%$ & LLLg & 102.80 & $0.003 \%$ & $0.000 \%$ \\
\hline OLEI & 64.10 & $7.790 \%$ & $0.254 \%$ & POA & 104.09 & $0.091 \%$ & $0.002 \%$ \\
\hline PLEI & 65.44 & $4.556 \%$ & $0.134 \%$ & SSO & 104.19 & $0.339 \%$ & $0.009 \%$ \\
\hline LLO & 66.33 & $1.018 \%$ & $0.020 \%$ & PBL & 104.37 & $0.012 \%$ & $0.000 \%$ \\
\hline PoPL & 66.95 & $0.008 \%$ & $0.001 \%$ & SLA & 104.45 & $0.034 \%$ & $0.001 \%$ \\
\hline LLP & 67.64 & $0.863 \%$ & $0.024 \%$ & ElEICe & 105.19 & $0.006 \%$ & $0.000 \%$ \\
\hline ElEIG & 68.65 & $1.274 \%$ & $0.038 \%$ & OL-23 & 105.83 & $0.002 \%$ & $0.000 \%$ \\
\hline EIEIS & 70.83 & $8.941 \%$ & $0.531 \%$ & OOB & 108.76 & $0.004 \%$ & $0.000 \%$ \\
\hline OOEI & 70.94 & $4.202 \%$ & $0.118 \%$ & OLLg & 108.88 & $0.008 \%$ & $0.001 \%$ \\
\hline POEI & 72.50 & $3.003 \%$ & $0.084 \%$ & PLLg & 110.44 & $0.006 \%$ & $0.000 \%$ \\
\hline LLG & 73.27 & $0.185 \%$ & $0.042 \%$ & SLB & 110.51 & $0.006 \%$ & $0.001 \%$ \\
\hline OOL & 73.53 & $1.782 \%$ & $0.048 \%$ & SOA & 110.62 & $0.028 \%$ & $0.001 \%$ \\
\hline PPEI & 74.35 & $0.429 \%$ & $0.014 \%$ & POB & 110.66 & $0.008 \%$ & $0.000 \%$ \\
\hline POPo & 74.40 & $0.041 \%$ & $0.002 \%$ & $00-23$ & 111.52 & $0.002 \%$ & $0.000 \%$ \\
\hline POL & 75.30 & $2.094 \%$ & $0.033 \%$ & OL-25 & 111.75 & $0.002 \%$ & $0.000 \%$ \\
\hline LLS & 76.15 & $0.484 \%$ & $0.012 \%$ & OOLg & 114.56 & $0.005 \%$ & $0.000 \%$ \\
\hline PPL & 77.15 & $0.701 \%$ & $0.012 \%$ & OLCe & 114.71 & $0.002 \%$ & $0.000 \%$ \\
\hline LEIA & 79.48 & $0.277 \%$ & $0.001 \%$ & POLg & 116.16 & $0.003 \%$ & $0.001 \%$ \\
\hline EIEIA & 79.89 & $0.474 \%$ & $0.010 \%$ & PLCe & 116.35 & $0.001 \%$ & $0.000 \%$ \\
\hline SOEI & 82.19 & $2.995 \%$ & $0.100 \%$ & SOB & 116.54 & $0.003 \%$ & $0.000 \%$ \\
\hline 000 & 82.21 & $0.961 \%$ & $0.018 \%$ & SLLg & 116.57 & $0.003 \%$ & $0.000 \%$ \\
\hline OLG & 82.29 & $0.342 \%$ & $0.051 \%$ & $00-25$ & 117.51 & $0.001 \%$ & $0.000 \%$ \\
\hline OOP & 84.24 & $1.413 \%$ & $0.030 \%$ & POCe & 122.29 & $0.002 \%$ & $0.001 \%$ \\
\hline EISP & 84.32 & $0.974 \%$ & $0.059 \%$ & SOLg & 122.81 & $0.004 \%$ & $0.002 \%$ \\
\hline PLG & 84.37 & $0.168 \%$ & $0.045 \%$ & Sum & & $100.000 \%$ & \\
\hline
\end{tabular}


Table S-23. Detailed triacylglycerol (TAG) composition of SBO by response factor normalized APCI-MS. TAG structures are not regiospecific.

\begin{tabular}{|c|c|c|c|c|c|c|c|}
\hline \multicolumn{8}{|c|}{ Soybean Oil } \\
\hline TAG & RT & \% Comp. & SD & TAG & RT & \% Comp. & SD \\
\hline $\operatorname{LnLnLn}$ & 49.69 & $0.068 \%$ & $0.002 \%$ & LLB & 95.03 & $0.319 \%$ & $0.004 \%$ \\
\hline LnLnL & 52.73 & $0.919 \%$ & $0.012 \%$ & POS & 95.97 & $0.560 \%$ & $0.009 \%$ \\
\hline LLLn & 56.28 & $6.383 \%$ & $0.026 \%$ & PLA & 96.53 & $0.239 \%$ & $0.007 \%$ \\
\hline LnLnO & 57.15 & $0.537 \%$ & $0.118 \%$ & SSL & 96.63 & $0.463 \%$ & $0.017 \%$ \\
\hline $\operatorname{Ln} \operatorname{Ln} P$ & 58.18 & $0.207 \%$ & $0.007 \%$ & LLnLg & 96.94 & $0.028 \%$ & $0.001 \%$ \\
\hline LLL & 60.32 & $16.528 \%$ & $0.303 \%$ & OL-21 & 98.69 & $0.022 \%$ & $0.000 \%$ \\
\hline OLLn & 61.32 & $4.870 \%$ & $0.130 \%$ & LL-23 & 99.14 & $0.027 \%$ & $0.001 \%$ \\
\hline PLLn & 62.55 & $3.175 \%$ & $0.097 \%$ & PPS & 99.59 & $0.009 \%$ & $0.000 \%$ \\
\hline LLO & 66.38 & $14.021 \%$ & $0.518 \%$ & OOA & 102.25 & $0.071 \%$ & $0.004 \%$ \\
\hline PoPL & 66.99 & $0.009 \%$ & $0.002 \%$ & OLB & 102.68 & $0.183 \%$ & $0.010 \%$ \\
\hline OOLn & 67.70 & $2.337 \%$ & $0.017 \%$ & LLLg & 102.91 & $0.093 \%$ & $0.005 \%$ \\
\hline LLP & 67.75 & $12.466 \%$ & $0.073 \%$ & SSO & 104.28 & $0.165 \%$ & $0.011 \%$ \\
\hline PoOL & 68.88 & $0.247 \%$ & $0.022 \%$ & POA & 104.32 & $0.092 \%$ & $0.006 \%$ \\
\hline POLn & 68.95 & $1.274 \%$ & $0.098 \%$ & PBL & 104.38 & $0.181 \%$ & $0.008 \%$ \\
\hline PPLn & 70.72 & $0.140 \%$ & $0.008 \%$ & SLA & 104.55 & $0.104 \%$ & $0.005 \%$ \\
\hline OOL & 73.79 & $5.986 \%$ & $0.094 \%$ & $00-21$ & 105.81 & $0.008 \%$ & $0.001 \%$ \\
\hline LLG & 73.89 & $0.262 \%$ & $0.010 \%$ & OL-23 & 106.05 & $0.015 \%$ & $0.000 \%$ \\
\hline POPO & 74.86 & $0.039 \%$ & $0.006 \%$ & LL-25 & 106.37 & $0.008 \%$ & $0.000 \%$ \\
\hline POL & 75.45 & $7.306 \%$ & $0.040 \%$ & OOB & 108.90 & $0.096 \%$ & $0.005 \%$ \\
\hline LLS & 76.37 & $4.198 \%$ & $0.048 \%$ & OLLg & 109.08 & $0.057 \%$ & $0.002 \%$ \\
\hline PPL & 77.28 & $2.301 \%$ & $0.041 \%$ & LLCe & 109.18 & $0.007 \%$ & $0.001 \%$ \\
\hline SOLn & 77.91 & $0.409 \%$ & $0.015 \%$ & РОВ & 110.57 & $0.055 \%$ & $0.003 \%$ \\
\hline $\operatorname{LLn} A$ & 78.93 & $0.057 \%$ & $0.005 \%$ & PLLg & 110.69 & $0.072 \%$ & $0.003 \%$ \\
\hline 000 & 82.51 & $2.809 \%$ & $0.131 \%$ & SOA & 110.81 & $0.031 \%$ & $0.002 \%$ \\
\hline OLG & 82.59 & $0.151 \%$ & $0.004 \%$ & SLB & 110.90 & $0.080 \%$ & $0.004 \%$ \\
\hline PLG & 84.47 & $0.131 \%$ & $0.020 \%$ & $00-23$ & 111.80 & $0.006 \%$ & $0.001 \%$ \\
\hline OOP & 84.47 & $2.620 \%$ & $0.100 \%$ & OL-25 & 111.99 & $0.005 \%$ & $0.000 \%$ \\
\hline SLO & 85.33 & $3.370 \%$ & $0.198 \%$ & OOLg & 114.74 & $0.031 \%$ & $0.001 \%$ \\
\hline LLA & 86.07 & $0.247 \%$ & $0.013 \%$ & OLCe & 114.79 & $0.005 \%$ & $0.001 \%$ \\
\hline POP & 86.45 & $0.641 \%$ & $0.041 \%$ & POLg & 116.40 & $0.015 \%$ & $0.003 \%$ \\
\hline SLP & 87.37 & $1.736 \%$ & $0.054 \%$ & PLCe & 116.41 & $0.004 \%$ & $0.000 \%$ \\
\hline $\operatorname{LLnB}$ & 88.32 & $0.091 \%$ & $0.005 \%$ & SLLg & 116.78 & $0.018 \%$ & $0.001 \%$ \\
\hline MOS & 89.92 & $0.006 \%$ & $0.001 \%$ & SOB & 116.86 & $0.026 \%$ & $0.002 \%$ \\
\hline PPP & 89.92 & $0.008 \%$ & $0.000 \%$ & $00-25$ & 117.68 & $0.002 \%$ & $0.000 \%$ \\
\hline LL-21 & 90.61 & $0.038 \%$ & $0.001 \%$ & OOCe & 120.50 & $0.002 \%$ & $0.000 \%$ \\
\hline OOG & 91.23 & $0.054 \%$ & $0.003 \%$ & POCe & 122.58 & $0.001 \%$ & $0.000 \%$ \\
\hline oos & 93.80 & $1.068 \%$ & $0.041 \%$ & SLCe & 122.66 & $0.001 \%$ & $0.000 \%$ \\
\hline LOA & 94.54 & $0.177 \%$ & $0.007 \%$ & SOLg & 122.86 & $0.007 \%$ & $0.000 \%$ \\
\hline
\end{tabular}




\section{9. ${ }^{2} \mathrm{D}$ UHPLC Plots for cherry pit oil.}

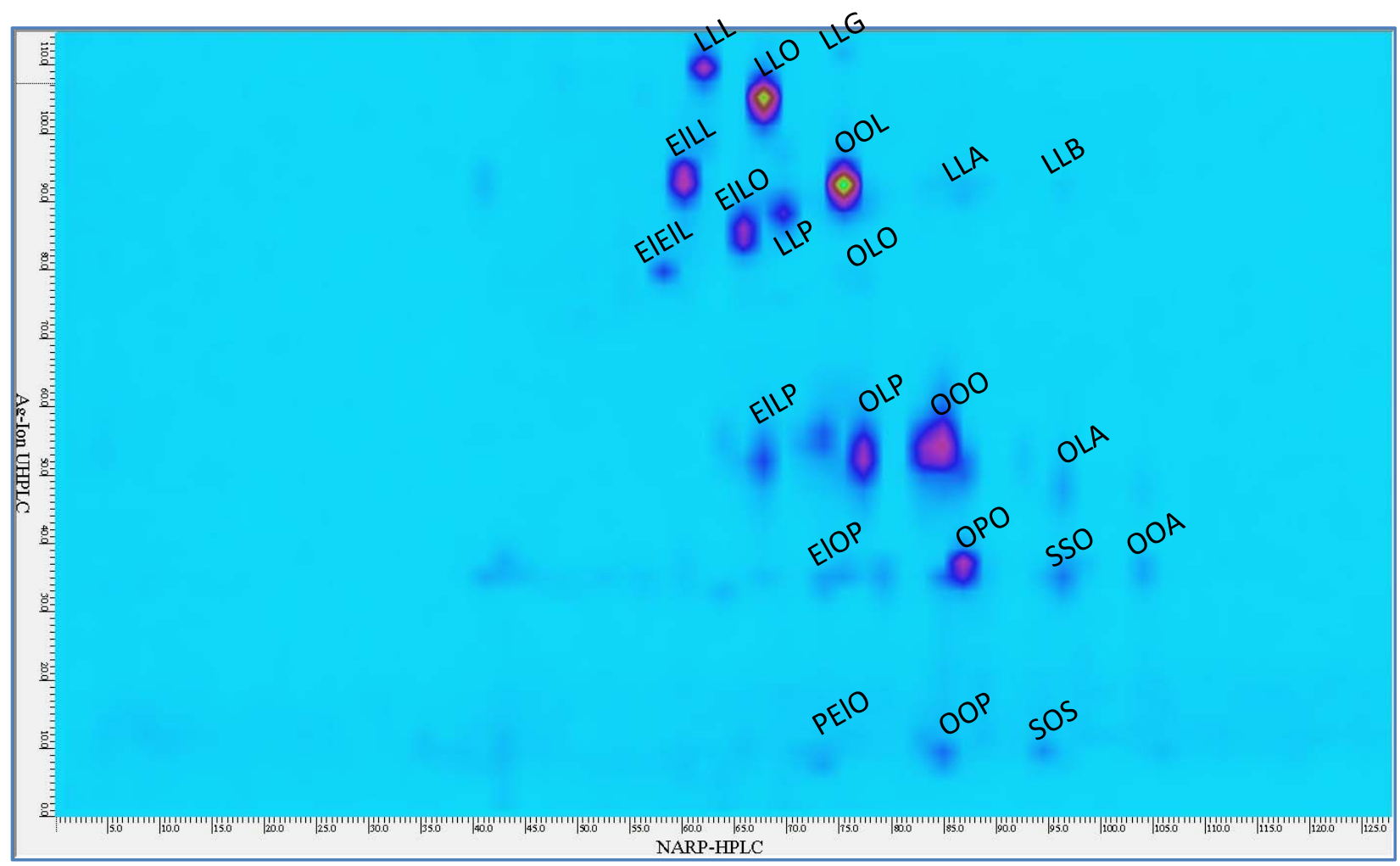

Figure S-4. ${ }^{2}$ D UHPLC contour plot of cherry pit oil by APPI-MS on TSQ Quantum Access Max.

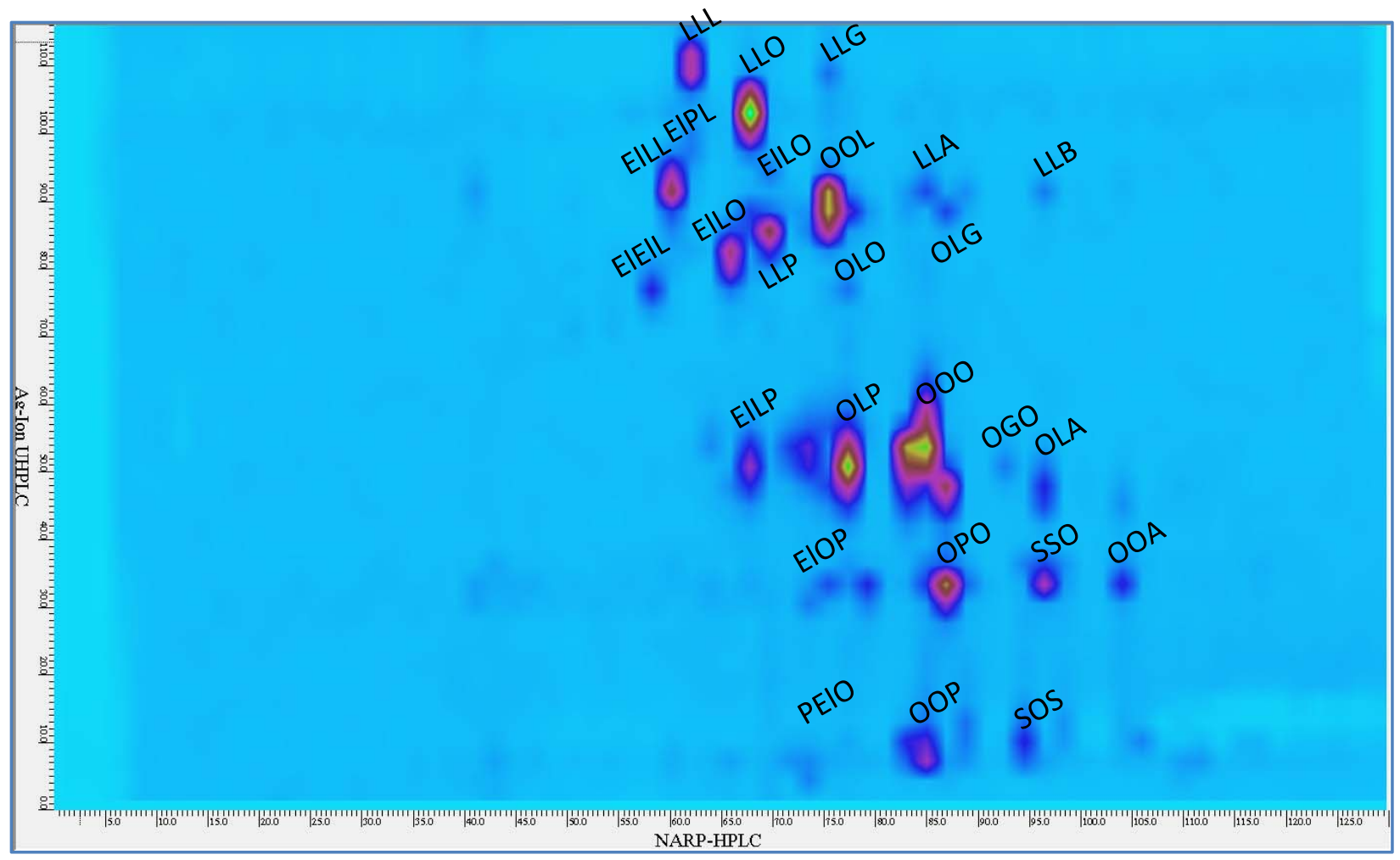

Figure S-5. ${ }^{2}$ D UHPLC contour plot of cherry pit oil by ESI-MS on LCQ Deca XP. 
10. ${ }^{2} \mathrm{D}$ UHPLC Plots for soybean oil.

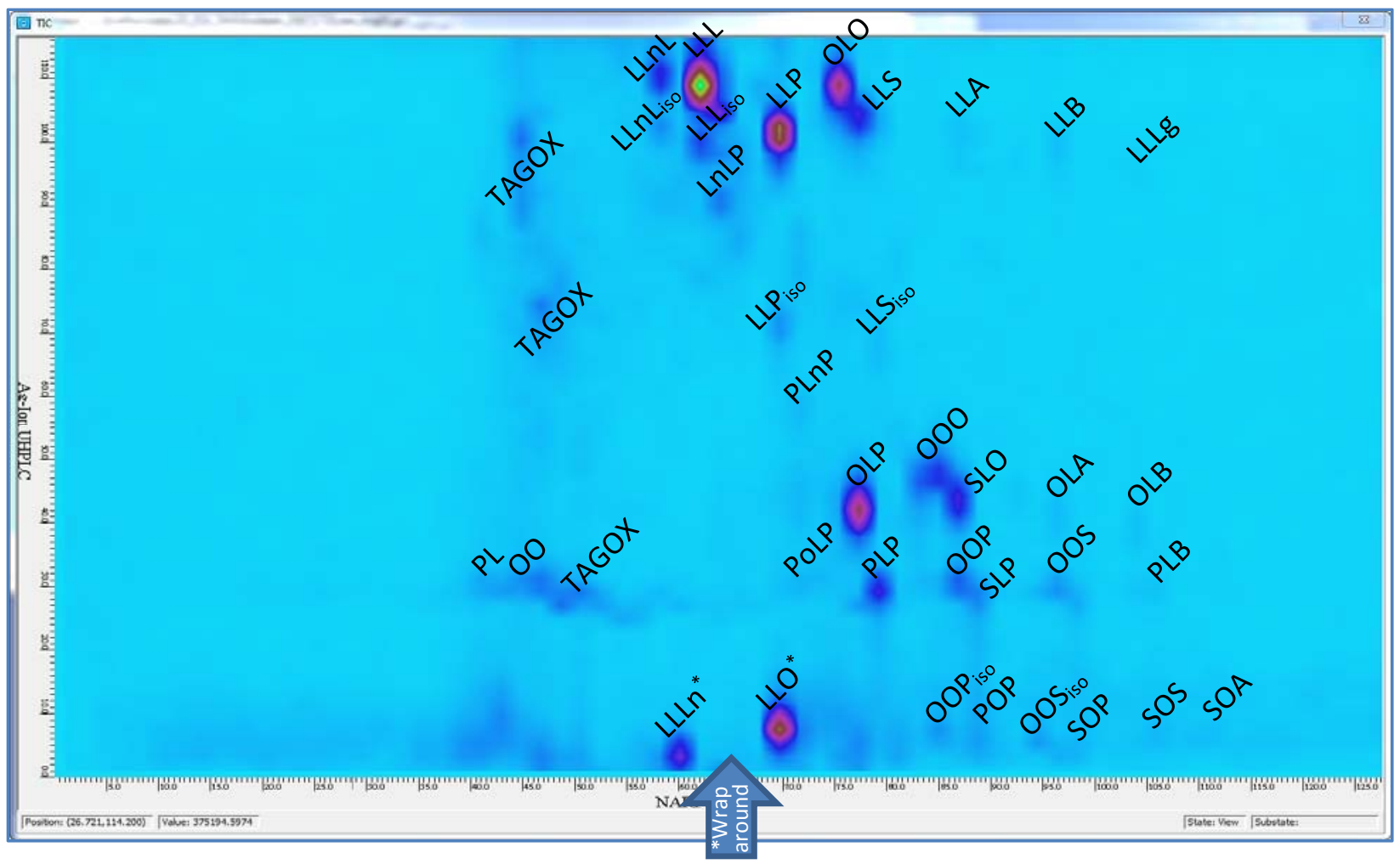

Figure S-6. ${ }^{2}$ D UHPLC contour plot of soybean oil by APPI-MS on TSQ Quantum Access Max.

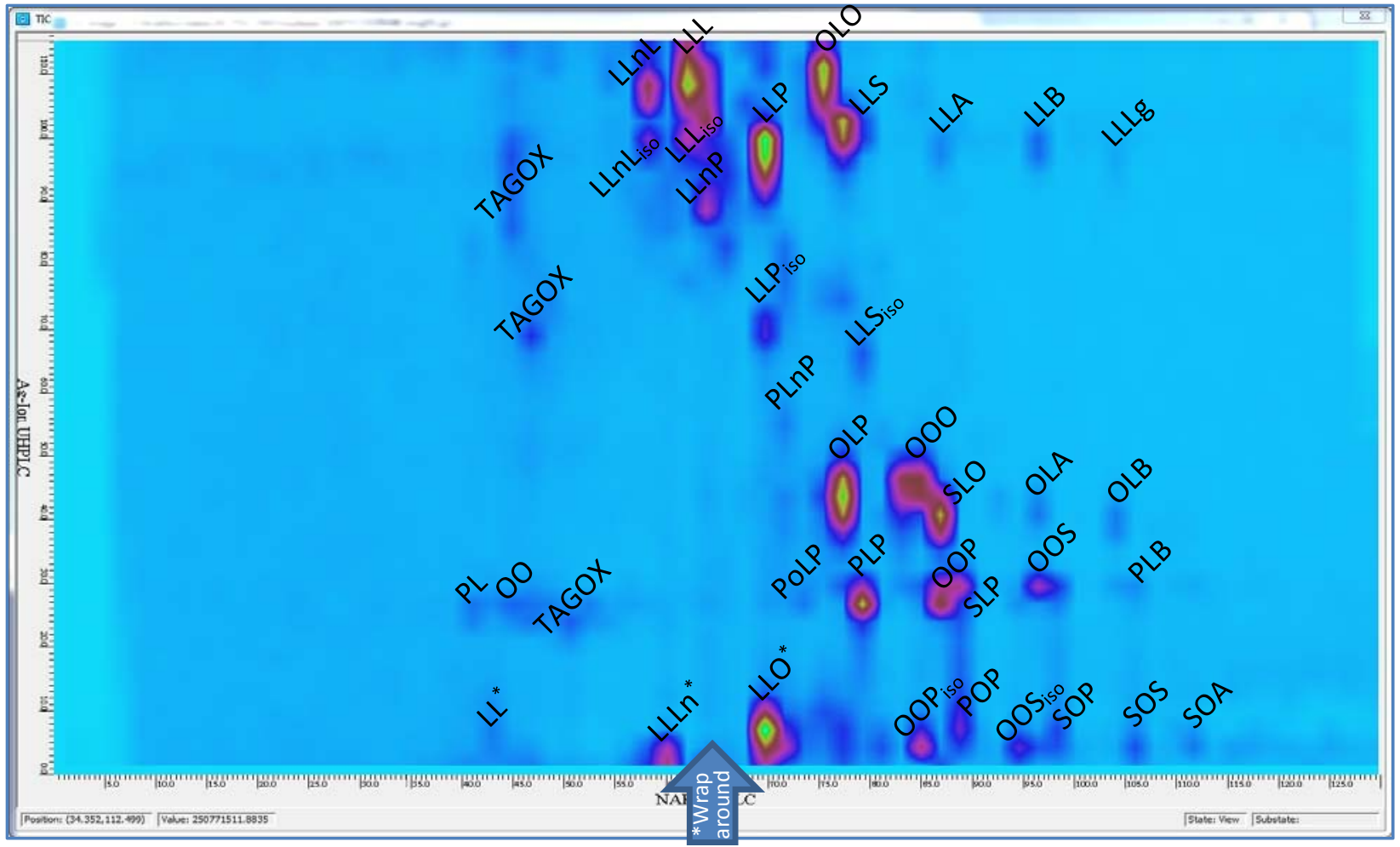

Figure S-7. ${ }^{2}$ D UHPLC contour plot of soybean oil by ESI-MS on LCQ Deca XP. 
Figures S-6 and S-7 show that TAGs with few degrees of unsaturation could be forced to be retained on the column longer by using a sharper reverse gradient, shown in Figure S-3. Notice the elution time of OLO in Figures S-6 and S-7 compared to OOL in Figures S-4 and S-5. However, LLLn and LLO experienced "wraparound" by eluting in the next modulation period, due to the lower ACN composition. Therefore, we have increased the ACN percentage to be intermediate between the gradient used for eleostearic acid-containing oils and this gradient. But we show these data to demonstrate the control that fine-tuning the ACN composition allows and because the quantification of the FSV and TAGs from the ${ }^{1} D$ was unaffected.

The TAG identities are given by the masses of the $\left[\mathrm{M}+\mathrm{NH}_{4}\right]^{+}$ions in ESI-MS spectra, and by $[\mathrm{M}+\mathrm{H}]^{+}$ and $[D A G]^{+}$fragments in APPI-MS spectra. As we reported previously, the [DAG] ${ }^{+}$fragment ratios in ESIMS and APPI-MS mass spectra are not as consistent for regioisomer identification as APCI-MS mass spectra. ${ }^{8}$ Therefore, regioisomers identities for SBO were taken from our earlier report of SBO isomers ${ }^{7}$ and were inferred from elution characteristics in the ${ }^{2}$ D UHPLC chromatograms, not solely from [DAG] ${ }^{+}$ ratios. APPI-MS has the distinct advantage that it is a non-contact ionization mode, unlike APCI-MS that accumulates a 'glob' of residue on the corona needle after extended exposure to solvents containing acetonitrile. But the [DAG] ${ }^{+}$fragment ratios are not as directly correlated with the regioisomeric positions of FAs in TAGs as in APCI-MS spectra. Thus, there is a trade-off in the use of APPI-MS versus APCI-MS.

The soybean oil analyzed for these experiments was a Halal SBO ordered from an online supplier, and showed higher levels of 18:3 isomers by GC-FID and GC-MS than most SBOs we have analyzed in the past, as well as some early-eluting TAG oxidation products (TAGOX). Normal linolenic acid represented $63.9 \%$ of all $18: 3$ species by GC-FID, another isomer was $15.8 \%$, a third was $15.0 \%$, and a fourth was $5.4 \%$. Linoleic acid also showed isomers, with normal linoleic acid being $96.0 \%$ by GC-FID, one isomer being $2.1 \%$ and another being $1.9 \%$. Two isomers of $18: 1$ were present, with oleic acid being $94.2 \%$ and the second isomer being 5.8\%. Thus, Figures S-6 and S-7 show some of the minor TAG peaks labelled with "iso" to indicate additional isomer peaks. The combination of oxidation products and isomers may indicate that the oil was not stored properly prior to sale, or may indicate sample production or processing issues. Based on the current status of literature precedent, the ESI-MS and APPI-MS mass spectra were not sufficiently definitive to allow localization of the double bond positions. Of course, with adequate standards, the $2 \mathrm{D}-\mathrm{LC}$ retention times could be used to identify the isomers, since, in many cases, they were separated on the new Ag-Ion UHPLC column. Since individual double bond isomers have not been specifically identified, the results for SBO FAs, DAGs, and TAGs in Tables S-17, S20 , and S-23, respectively, represent the sum of all isomers for each FA.

\section{Supporting Information References}

(1) Folch, J.; Lees, M.; Sloane-Stanley, G. H. J. Biol. Chem. 1957, 226, 497-509.

(2) Korlesky, N. M.; Stolp, L. J.; Kodali, D. R.; Goldschmidt, R.; Byrdwell, W. C. J. Am. Oil Chem. Soc. 2016, 1-11.

(3) Slover, H. T.; Thompson, R. H.; Merola, G. V. J. Am. Oil Chem. Soc. 1983, 60, 1524-1528.

(4) Matthäus, B.; Özcan, M. M. J. Food Lipids 2009, 16, 187-199.

(5) Sheng, J.; Vannela, R.; Rittmann, B. E. Bioresour. Technol. 2011, 102, 1697-1703.

(6) Byrdwell, W. C. J. Agric. Food Chem. 2009, 57, 2135-2146.

(7) Byrdwell, W. C. Anal. Bioanal. Chem. 2015, 407, 5143-5160.

(8) Byrdwell, W. C. J. Am. Oil Chem. Soc. 2015, 92, 1533-1547. 